\title{
Light and Motion in the Local Volume
}

\author{
Alan B. Whiting \\ Cerro Tololo Inter-American Observatory \\ Casilla 603, La Serena, Chile \\ awhiting@noao.edu
}

\begin{abstract}
Using high-quality data on 149 galaxies within 10 megaparsecs (Mpc), I find no correlation between luminosity and peculiar velocity at all. There is no unequivocal sign on scales of 1-2 Mpc of the expected gravitational effect of the brightest galaxies, in particular infall toward groups; or of infall toward the Supergalactic Plane on any scale. Either dark matter is not distributed in the same way as luminous matter in this region, or peculiar velocities are not due to fluctuations in mass. The sensitivity of peculiar velocity studies to the background model is highlighted.
\end{abstract}

Subject headings: galaxies: kinematics and dynamics - dark matter - large-scale structure of the universe

\section{Introduction}

The first subject of cosmology is the overall motion of the universe. In terms of the current understanding, this means figuring out whether it is open or closed, flat or curved, dense or rarified; and determining a few numbers $\left(H_{0}, \Omega\right)$ which describe it. In the past few years this task seems to have been carried almost to completion.

The next task is to study the details of the universe, that is, the departures from smoothness and overall motion. These take the form of the visible features (galaxies, as well as collections of them in groups, clusters, superclusters, and so forth) and their peculiar motions. On the largest scales this may be approached by using perturbations of the background cosmology, where linearity makes the calculations easier (or possible at all). Here, the picture of structure growing by gravitational instability from small initial fluctuations in a smooth distribution of dark and luminous matter seems to work well (see Courteau, Strauss, \& Willick (2000) for a review; more recent work includes Branchini et al. (2000) 
and Nusser et al. (2001)). There are disagreements in detail, for instance over the size of the bias parameter between dark and luminous matter, but the overall picture shows no great difficulties.

The problem of the formation of individual galaxies is much complicated by the inclusion of radiation and gas effects, and it is beyond the scope of this paper even to outline the subfield. For our purposes it suffices to note that dark matter and luminous matter are not distributed the same way on galactic scales, the dark halo being larger than any directly detectable part of a galaxy, though the two parts appear to be concentric.

On scales smaller than large-scale structure and larger than galaxies we then expect to see a transition. Dark matter should depart significantly from luminous matter in its distribution, while retaining a similar concentration around the densest points. Exactly how this happens should tell us something about dark matter itself.

Unfortunately, studying structures in which the density contrast is highly nonlinear is mathematically difficult. One approach is to use galaxy clusters, which should be virialized (or nearly so). Another is to find a region in which observational data are abundant and of high quality, and in which peculiar velocities indicate dynamical youth. Here the motions should be simpler than in a dynamically old system, while at the same time even small motions should be visible. Such a region is the Local Volume, within about 10 megaparsecs (Mpc). In the Volume there are radial velocities accurate to one or a few $\mathrm{km} \mathrm{s}^{-1}$ for hundreds of galaxies, and (recently) distances good to $10 \%$ or better for a large fraction. Using these data, the present study aims to compare the distribution and motion of luminous matter, in an effort to show something of the location of gravitating matter.

\section{Data}

\subsection{Sources and Treatment}

Data for 149 galaxies in the Local Volume were collected from the literature and are presented in Table (1) (ordered by Supergalactic longitude $L$, as are the rest of the data tables in this paper). The main criterion for inclusion was a distance known to $10 \%$ or better, along with a reliable radial velocity.

The criterion for distance uncertainty was rather restrictive, and in fact it is possible to derive some kinematic or dynamic properties of the Volume with a larger set of poorer data (as done in, for instance, Whiting (2003) or Karachentsev \& Makarov (2001), or any work on large-scale structure). But the focus here is on the details of the peculiar velocity 
field, for which a finer resolution is needed. A galaxy with a distance uncertainty of $10 \%$ at $10 \mathrm{Mpc}$ gives a peculiar radial velocity uncertainty of something over $60 \mathrm{~km} \mathrm{~s}^{-1}$, which is about the largest which could be tolerated here.

This means that some popular methods, such as the Tully-Fisher relation, are not useful, and in fact almost all distances were obtained using Cepheid variables (Ceph in the table) or the brightness of the tip of the Red Giant Branch as found in a $I, V-I$ color-magnitude diagram (TRGB). Some variations on the latter use different filters ( $K$, the SDSS system), or a slightly different calibration for the absolute magnitude of the tip $\left(M_{I}=-4.05\right.$ instead of -4.00); they are marked where they occur, and any resulting differences in distance lie within the quoted errors.

The technique of surface brightness fluctuation (SBF) seems quite promising and should have added a few more objects. However, the SBF distances for DDO 181 (Hidalgo et al. 2003) and ESO 540-32 (Jerjen et al. 1998) disagree rather strongly with the TRGB distances (Karachentsev et al. (2002c) and Jerejen \& Rejkuba (2001), respectively), and NGC 4736 is not much better (Tonry et al. (2001) and Karachentsev et al. (2003a)). Since the number of galaxies with only SBF distances is small, it was felt better to leave them out rather than add a source of unknown error.

Where more than one distance estimate was available, and all were consistent, they were combined. If they were within their stated relative errors, they were averaged and the uncertainty reduced by a factor of $\sqrt{N}$; if they were between one and two uncertainties distant, they were averaged and the larger uncertainty taken. If they were not reconcilable (for instance, with IC 10) a judgement as to the more reliable distance(s) was made.

Even though 149 is a gratifying number of galaxies to work with (being more than four times the number of good data-points used in a previous calculation, Whiting (2003)), it still does not include half of the total number present; nor, more importantly, are all the brightest galaxies there. To include all galaxies which have been known or suspected to be brighter than $M_{B} \sim 18.5$ and in the Local Volume, 21 additional objects are presented in Table (2). The methods used in several of these cases might in fact give high-quality data, since such things as the brightest-star method and SBF seem to work satisfactorily with larger galaxies. This needs to be checked, though, and Table (2) could usefully be taken as a target list for a deep TRGB observing program.

Brightest-star distances are assigned an uncertainty of 0.4 magnitude. Distances to the major Sculptor Group galaxies were determined by Puche \& Carignan (1988) from a variety of methods; as those determined since for NGC 300, 253 and 7793 are systematically larger, those for NGC 55 and 247 have been adjusted accordingly (note that all of these are within 
the uncertainties given in the 1988 paper). Distances derived solely from radial velocities use the kinematic isotropic-expansion model derived later in the present paper; uncertainties are figured as the peculiar radial velocity dispersion times the effective Hubble constant.

Apparent $B$ magnitudes are derived from $\mathrm{NED}^{1}$, and are available for almost all objects. However, most of these numbers come from relatively inaccurate photographic photometry; for later calculations they are all assigned an uncertainty of 0.4 magnitude. Almost all of the $K$ photometry is from the large, uniform and well-controlled data resulting from the 2MASS survey (here conveniently extracted also via NED). It is of course preferable to use better data where they exist, and for questions of mass-to-light ratios to use near infrared rather than the much more variable $B$; but less than half of the objects have $K$ measurements. For most of the following, calculations with be done in both bands, investigating the effects of sample completeness against photometric accuracy (and assumed closeness to the actual mass field). Absolute magnitudes for those dwarf galaxies without photometry are arbitrarily set at -10 (in $B$ or $K$ ); these should have no effect on the results, while avoiding troublesome zeros in calculations. Galactic absorption is taken from Schlegel et al. (1998) by way of NED except in the case of the Circinus galaxy, where strong and variable absorption meant that the separate determination in Freeman et al. (1977) had to be used.

\subsection{Notes on Particular Objects}

IC 10:

A distance modulus of $24.9 \pm .2$ is found in Hunter (2001), which is at variance with the others used here. Extinction is a large factor in the disagreement.

DDO 18\%:

The Cepheid distance of Hoessel et al. (1998b) is almost three times the TRGB distance here adopted; this was attributed by Aparicio et al. (2000) to problems with analyzing a small number of variable stars.

$L M C$ :

Because the major methods of distance determination (Cepheids and TRGB) are calibrated to an LMC distance of $18.50(50 \mathrm{kpc})$, it is here fixed at that distance.

$L M C$ and $S M C$ :

Neither of these has $K$-band photometry, for reasons which are obvious on reflection.

\footnotetext{
${ }^{1}$ NED is the NASA/IPAC Extragalactic Database, which is operated by the Jet Propulsion Laboratory, California Institute of Technology, under contract with the National Aeronautics and Space Administration.
} 
They are in fact brighter than most of the galaxies in the sample and so by rights should be included somehow. But since they are so close to the Milky Way, their $K$ luminosities do not affect any calculations separately from it; and their luminosities are almost certainly much smaller than the uncertainty in the Milky Way's. They are therefore ignored in the $K$-band calculations.

Phoenix:

There are two mutually incompatible optical radial velocity measurements for Phoenix, $-52 \pm 6$ (Gallart et al. 2001) and $-13 \pm 9$ (Irwin \& Tolstoy 2002). There is no obvious explanation for the difference between them, nor any clear reason to choose either. I have (somewhat arbitrarily) used the latter.

$P G C$ 20125 and PGC 39032:

These had no listed radial velocity uncertainty, and the original measurements could not be traced. $10 \mathrm{~km} \mathrm{~s}^{-1}$ is a conservative educated guess.

\section{Tucana:}

This also had no listed radial velocity uncertainty; the bins in the paper are $10 \mathrm{~km} \mathrm{~s}^{-1}$ wide, so this is a reasonable amount.

\section{Carina:}

The listed photometry is taken from Matero et al. (1993), and is based on model-fitting. Milky Way:

The apparent magnitudes are back-calculated from the absolute magnitudes listed in Suntzeff et al. (1992); the uncertainties are guesses.

\section{NGC 1569:}

This is assigned the shorter (and slightly more probable) TRGB distance from Makarova \& Karachentsev (2003). Due to the uncertainty over which is the better figure, however, it is not included in the table of better-quality data.

\section{NGC 5194 and 5195:}

NGC 5195, the smaller part of M 51, has an independent (SBF) distance; there appears to be no separate determination for NGC 5194, at least of moderate to high quality. I have assigned them the same distance. Normally I strenuously avoid anything like this "companion" or "member of the same group" argument for distance; but in this case there appears to be no choice. Note that NGC 5194 is not used in any peculiar velocity calculations, only in those of the luminosity field.

KKH96, KK98-34, KK98-41, NGC 1560 and KK98-44:

There is no radial velocity error listed for these in the reference. An estimate of $5 \mathrm{~km}$ 
$\mathrm{s}^{-1}$ is here adopted. 
Table 1. Local Volume Data

\begin{tabular}{|c|c|c|c|c|c|c|c|c|c|c|c|c|c|c|}
\hline Name & $\begin{array}{c}\mathrm{L} \\
\text { Degrees }\end{array}$ & $\begin{array}{c}\text { B } \\
\text { Degrees }\end{array}$ & $\begin{array}{c}\mathrm{RV} \\
\mathrm{km} \mathrm{s}^{-1}\end{array}$ & $\begin{array}{c}\Delta \mathrm{RV} \\
\mathrm{km} \mathrm{s}^{-1}\end{array}$ & RV Source & $\begin{array}{c}\mathrm{D} \\
\mathrm{Mpc}\end{array}$ & $\begin{array}{l}\Delta \mathrm{D} \\
\mathrm{Mpc}\end{array}$ & D Source & D Method & Absorption & B Mag & K Mag & $\Delta \mathrm{K}$ Mag & K Mag Source \\
\hline KK98-35 & 10.33 & 0.32 & -66.0 & 5.0 & 1 & 3.16 & 0.32 & 1 & TRGB405 & 2.339 & 17.20 & $\ldots$ & . & $\ldots$ \\
\hline IC342 & 10.60 & 0.37 & 31.0 & 3.0 & NED & 3.28 & 0.27 & 2 & Ceph & 2.407 & 9.10 & 4.56 & 0.04 & $2 \mathrm{M}$ \\
\hline UGCA105 & 15.00 & -9.25 & 111.0 & 5.0 & NED & 3.15 & 0.32 & 3 & TRGB & 1.351 & 13.90 & $\ldots$ & $\ldots$ & $\ldots$ \\
\hline KK98-44 & 15.04 & -4.21 & 77.0 & 5.0 & 1 & 3.34 & 0.00 & 1 & TRGB405 & 0.936 & 16.10 & $\ldots$ & $\ldots$ & $\ldots$ \\
\hline NGC1560 & 16.03 & 0.79 & -36.0 & 5.0 & 1 & 3.45 & 0.37 & 1 & TRGB405 & 0.812 & 12.16 & 8.93 & 0.03 & $2 \mathrm{M}$ \\
\hline KK98-41 & 16.09 & 1.87 & -47.0 & 5.0 & 1 & 3.93 & 0.47 & 1 & TRGB405 & 0.951 & 14.84 & $\ldots$ & $\ldots$ & $\ldots$ \\
\hline ККН34 & 22.54 & -0.41 & 110.0 & 2.0 & NED & 4.61 & 0.36 & 4 & TRGB405 & 1.076 & 17.10 & $\ldots$ & $\ldots$ & $\ldots$ \\
\hline NGC6789 & 23.28 & 41.59 & -141.0 & 9.0 & NED & 3.63 & 0.37 & 5 & TRGB & 0.302 & 13.76 & 12.24 & 0.09 & $2 \mathrm{M}$ \\
\hline NGC2366 & 29.46 & -4.86 & 100.0 & 3.0 & NED & 3.19 & 0.41 & 6 & TRGB405 & 0.157 & 11.43 & 10.62 & 0.13 & $2 \mathrm{M}$ \\
\hline NGC2403 & 30.80 & -8.31 & 131.0 & 3.0 & NED & 3.16 & 0.25 & 7,8 & TRGBsdss,Ceph & 0.172 & 8.93 & 6.19 & 0.04 & $2 \mathrm{M}$ \\
\hline NGC6503 & 33.14 & 34.63 & 60.0 & 7.0 & NED & 5.27 & 0.77 & 4 & TRGB405 & 0.138 & 10.91 & 7.30 & 0.02 & $2 \mathrm{M}$ \\
\hline DDO50 & 33.26 & -2.36 & 157.0 & 1.0 & NED & 3.31 & 0.15 & 6,9 & TRGB405,Ceph & 0.139 & 11.10 & 8.79 & 0.04 & $2 \mathrm{M}$ \\
\hline KDG52 & 33.52 & -1.93 & 113.0 & 5.0 & NED & 3.55 & 0.26 & 6 & TRGB405 & 0.091 & 16.50 & $\ldots$ & $\ldots$ & $\ldots$ \\
\hline UGC4483 & 35.02 & -2.65 & 156.0 & 5.0 & NED & 3.29 & 0.13 & 10,11 & TRGB & 0.140 & 15.12 & $\ldots$ & $\ldots$ & $\cdots$ \\
\hline DDO53 & 36.25 & -6.04 & 19.0 & 10.0 & NED & 3.56 & 0.25 & 6 & TRGB405 & 0.160 & 14.48 & $\ldots$ & $\ldots$ & 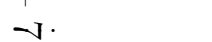 \\
\hline UGC3755 & 36.82 & -63.36 & 314.0 & 4.0 & NED & 5.10 & 0.27 & 12,4 & TRGB & 0.384 & 14.10 & $\ldots$ & $\ldots$ & ... \\
\hline VIIZw403 & 36.87 & 11.40 & -100.0 & 1.0 & NED & 4.41 & 0.10 & 12,13 & TRGB & 0.155 & 14.50 & 12.60 & 0.10 & 14 \\
\hline HoI & 38.77 & 1.33 & 143.0 & 1.0 & NED & 3.84 & 0.46 & 6 & TRGB405 & 0.207 & 13.00 & $\ldots$ & $\ldots$ & $\ldots$ \\
\hline M82 & 40.72 & 1.05 & 203.0 & 4.0 & NED & 3.89 & 0.38 & 15 & TRGB & 0.685 & 9.30 & 4.66 & 0.01 & $2 \mathrm{M}$ \\
\hline BK3N & 41.07 & 0.41 & -40.0 & 0.0 & NED & 4.29 & 0.26 & 6 & TRGB405 & 0.385 & 17.10 & $\ldots$ & $\ldots$ & $\ldots$ \\
\hline M81 & 41.12 & 0.59 & -34.0 & 4.0 & NED & 3.55 & 0.13 & 8 & Ceph & 0.346 & 7.89 & 3.83 & 0.02 & $2 \mathrm{M}$ \\
\hline NGC2976 & 41.33 & -0.78 & 3.0 & 5.0 & NED & 3.56 & 0.38 & 6 & TRGB405 & 0.300 & 10.82 & 7.52 & 0.03 & $2 \mathrm{M}$ \\
\hline KK98-81 & 41.54 & 0.33 & -135.0 & 30.0 & NED & 3.60 & 0.25 & 16 & TRGB & 0.309 & 15.60 & $\ldots$ & $\ldots$ & $\ldots$ \\
\hline NGC3077 & 41.85 & 0.83 & 14.0 & 4.0 & NED & 3.85 & 0.37 & 17 & TRGB & 0.289 & 10.61 & 7.30 & 0.02 & $2 \mathrm{M}$ \\
\hline Garland & 41.91 & 0.83 & 50.0 & 0.0 & NED & 3.82 & 0.42 & 6 & TRGB405 & 0.289 & 0.00 & $\ldots$ & $\ldots$ & $\ldots$ \\
\hline DDO82 & 42.04 & 3.85 & 180.0 & 60.0 & NED & 4.00 & 0.41 & 6 & TRGB405 & 0.188 & 13.47 & $\ldots$ & $\ldots$ & $\ldots$ \\
\hline KK98-85 & 42.74 & 0.44 & -18.0 & 14.0 & NED & 3.70 & 0.26 & 16 & TRGB & 0.235 & 18.00 & $\ldots$ & $\ldots$ & $\ldots$ \\
\hline DDO71 & 43.51 & -0.58 & -126.0 & 5.0 & NED & 3.50 & 0.24 & 16 & TRGB & 0.412 & 18.00 & $\ldots$ & $\ldots$ & $\ldots$ \\
\hline IC2574 & 43.63 & 2.31 & 57.0 & 2.0 & NED & 4.02 & 0.42 & 6 & TRGB405 & 0.156 & 10.80 & 10.72 & 0.10 & $2 \mathrm{M}$ \\
\hline Draco & 43.79 & 44.23 & -292.0 & 21.0 & NED & 0.76 & 0.01 & 18 & RRL & 0.118 & 10.90 & $\ldots$ & $\ldots$ & $\ldots$ \\
\hline KDG73 & 44.03 & 4.75 & -132.0 & 6.0 & NED & 3.82 & 0.23 & 6 & TRGB405 & 0.080 & 14.90 & $\ldots$ & $\ldots$ & $\ldots$ \\
\hline DDO78 & 44.10 & 1.69 & 55.0 & 10.0 & 19 & 3.72 & 0.26 & 16 & TRGB & 0.094 & 15.80 & $\ldots$ & $\ldots$ & $\ldots$ \\
\hline DDO47 & 46.54 & -55.49 & 272.0 & 1.0 & NED & 5.18 & 0.60 & 4 & TRGB405 & 0.145 & 13.60 & $\ldots$ & $\ldots$ & $\ldots$ \\
\hline KK98-65 & 46.93 & -55.67 & 407.0 & 10.0 & 20 & 4.51 & 0.37 & 4 & TRGB405 & 0.137 & 15.60 & $\ldots$ & $\ldots$ & \\
\hline
\end{tabular}


Table 1-Continued

\begin{tabular}{|c|c|c|c|c|c|c|c|c|c|c|c|c|c|c|}
\hline Name & $\begin{array}{c}\mathrm{L} \\
\text { Degrees }\end{array}$ & $\begin{array}{c}\text { B } \\
\text { Degrees }\end{array}$ & $\begin{array}{c}\mathrm{RV} \\
\mathrm{km} \mathrm{s}^{-1}\end{array}$ & $\begin{array}{c}\Delta \mathrm{RV} \\
\mathrm{km} \mathrm{s}^{-1}\end{array}$ & RV Source & $\begin{array}{c}\mathrm{D} \\
\mathrm{Mpc}\end{array}$ & $\begin{array}{l}\Delta \mathrm{D} \\
\mathrm{Mpc}\end{array}$ & D Source & D Method & Absorption & B Mag & K Mag & $\Delta \mathrm{K}$ Mag & K Mag Source \\
\hline NGC4236 & 47.11 & 11.38 & 0.0 & 4.0 & NED & 4.45 & 0.45 & 6 & TRGB405 & 0.063 & 10.05 & 9.01 & 0.05 & $2 \mathrm{M}$ \\
\hline UMi & 47.71 & 27.10 & -247.0 & 1.0 & NED & 0.08 & 0.00 & 21 & CMD & 0.137 & 11.90 & $\ldots$ & $\ldots$ & $\ldots$ \\
\hline DDO165 & 49.61 & 15.58 & 37.0 & 8.0 & NED & 4.57 & 0.40 & 6 & TRGB405 & 0.104 & 12.80 & $\ldots$ & $\ldots$ & $\ldots$ \\
\hline UGC4115 & 54.21 & -56.22 & 338.0 & 5.0 & NED & 5.50 & 0.58 & 4 & TRGB405 & 0.122 & 15.20 & $\ldots$ & $\ldots$ & $\ldots$ \\
\hline KKR25 & 56.09 & 40.37 & -135.0 & 2.0 & 22 & 1.86 & 0.12 & 23 & TRGB403 & 0.036 & 17.00 & $\ldots$ & $\ldots$ & $\ldots$ \\
\hline NGC5204 & 59.40 & 17.85 & 201.0 & 1.0 & NED & 4.65 & 0.53 & 24 & TRGB & 0.054 & 11.73 & 9.51 & 0.06 & $2 \mathrm{M}$ \\
\hline NGC3738 & 59.57 & 1.79 & 229.0 & 4.0 & NED & 4.90 & 0.54 & 24 & TRGB & 0.045 & 12.13 & $\ldots$ & $\ldots$ & $\ldots$ \\
\hline UGC8508 & 63.09 & 17.91 & 62.0 & 5.0 & NED & 2.56 & 0.15 & 3 & TRGB & 0.064 & 14.40 & $\ldots$ & $\ldots$ & $\ldots$ \\
\hline M101 & 63.58 & 22.61 & 241.0 & 2.0 & NED & 6.83 & 0.31 & 25,8 & Ceph & 0.037 & 8.31 & 5.51 & 0.05 & $2 \mathrm{M}$ \\
\hline UGC7298 & 63.89 & 6.63 & 172.0 & 5.0 & NED & 4.21 & 0.32 & 24 & TRGB & 0.098 & 15.00 & $\ldots$ & $\ldots$ & $\ldots$ \\
\hline UGC6541 & 64.19 & -0.79 & 249.0 & 4.0 & NED & 3.89 & 0.47 & 24 & TRGB & 0.078 & 0.00 & $\ldots$ & $\ldots$ & $\ldots$ \\
\hline NGC3741 & 67.96 & -2.08 & 229.0 & 4.0 & NED & 3.03 & 0.33 & 24 & TRGB & 0.110 & 14.30 & .. & $\ldots$ & $\ldots$ \\
\hline M106 & 68.74 & 5.55 & 448.0 & 3.0 & NED & 7.47 & 0.28 & 8,26 & Ceph,Geo & 0.069 & 9.10 & 5.46 & 0.02 & $2 \mathrm{M}$ \\
\hline LeoA & 69.91 & -25.80 & 20.0 & 4.0 & NED & 0.74 & 0.06 & 27,28 & CMD,RRL & 0.089 & 12.92 & $\ldots$ & $\ldots$ & $\ldots$ \\
\hline KK98-109 & 70.24 & -0.92 & 225.0 & 10.0 & 20 & 4.51 & 0.34 & 24 & TRGB & 0.083 & 17.50 & $\ldots$ & $\ldots$ & $\cdots \infty$ \\
\hline DDO167 & 71.75 & 14.42 & 164.0 & 6.0 & NED & 4.19 & 0.47 & 24 & TRGB & 0.041 & 17.00 & $\ldots$ & $\ldots$ & $\ldots 1$ \\
\hline DDO168 & 72.19 & 14.55 & 192.0 & 1.0 & NED & 4.33 & 0.49 & 24 & TRGB & 0.065 & 12.70 & $\ldots$ & $\ldots$ & $\ldots$ \\
\hline NGC4449 & 72.30 & 6.18 & 207.0 & 4.0 & NED & 4.21 & 0.50 & 24 & TRGB & 0.083 & 9.99 & 7.25 & 0.04 & $2 \mathrm{M}$ \\
\hline DDO125 & 72.85 & 5.93 & 196.0 & 4.0 & NED & 2.51 & 0.11 & 3,12 & TRGB & 0.088 & 12.84 & $\ldots$ & $\ldots$ & $\ldots$ \\
\hline DDO190 & 74.07 & 26.85 & 150.0 & 4.0 & NED & 2.85 & 0.16 & 3,29 & TRGB & 0.052 & 13.25 & $\ldots$ & $\ldots$ & $\ldots$ \\
\hline DDO99 & 74.93 & -2.12 & 242.0 & 1.0 & NED & 2.64 & 0.21 & 3 & TRGB & 0.113 & 13.40 & $\ldots$ & $\ldots$ & $\ldots$ \\
\hline M94 & 76.24 & 9.50 & 308.0 & 1.0 & NED & 4.66 & 0.59 & 24 & TRGB & 0.076 & 8.99 & 5.11 & 0.02 & $2 \mathrm{M}$ \\
\hline NGC4244 & 77.73 & 2.41 & 243.0 & 4.0 & NED & 4.49 & 0.47 & 24 & TRGB & 0.090 & 10.88 & 7.72 & 0.05 & $2 \mathrm{M}$ \\
\hline UGCA290 & 77.94 & 6.41 & 445.0 & 9.0 & NED & 6.61 & 0.37 & 30 & TRGB & 0.060 & 0.00 & $\ldots$ & $\ldots$ & $\ldots$ \\
\hline DDO181 & 78.09 & 18.58 & 201.0 & 1.0 & NED & 3.01 & 0.29 & 3 & TRGB & 0.026 & 14.70 & $\ldots$ & $\ldots$ & $\ldots$ \\
\hline IC3687 & 78.42 & 7.27 & 354.0 & 1.0 & NED & 4.57 & 0.48 & 24 & TRGB & 0.088 & 13.71 & $\ldots$ & $\ldots$ & $\ldots$ \\
\hline DDO126 & 78.93 & 4.03 & 218.0 & 5.0 & NED & 4.87 & 0.55 & 24 & TRGB & 0.060 & 14.20 & $\ldots$ & $\ldots$ & $\ldots$ \\
\hline NGC4214 & 79.02 & 1.60 & 291.0 & 3.0 & NED & 2.82 & 0.17 & 31,32 & TRGB & 0.094 & 10.24 & 7.91 & 0.05 & $2 \mathrm{M}$ \\
\hline DDO113 & 79.08 & 1.43 & 284.0 & 6.0 & NED & 2.86 & 0.14 & 3 & TRGB & 0.090 & 15.40 & $\ldots$ & $\ldots$ & $\ldots$ \\
\hline IC4182 & 80.34 & 11.61 & 321.0 & 1.0 & NED & 4.53 & 0.13 & 8 & Ceph & 0.059 & 13.00 & $\ldots$ & $\ldots$ & $\ldots$ \\
\hline UGC7605 & 80.39 & 3.92 & 309.0 & 5.0 & NED & 4.43 & 0.53 & 24 & TRGB & 0.063 & 15.00 & $\ldots$ & $\ldots$ & $\ldots$ \\
\hline NGC4395 & 82.31 & 2.73 & 319.0 & 1.0 & NED & 4.46 & 0.34 & 24,33 & TRGB, Ceph & 0.074 & 10.64 & 9.98 & 0.06 & $2 \mathrm{M}$ \\
\hline UGC8833 & 83.54 & 21.09 & 228.0 & 5.0 & NED & 3.19 & 0.21 & 24 & TRGB & 0.051 & 16.50 & $\ldots$ & $\ldots$ & $\ldots$ \\
\hline LeoI & 88.90 & -34.56 & 285.0 & 2.0 & NED & 0.26 & 0.01 & 12 & TRGB & 0.156 & 11.18 & $\cdots$ & $\cdots$ & $\cdots$ \\
\hline
\end{tabular}


Table 1-Continued

\begin{tabular}{|c|c|c|c|c|c|c|c|c|c|c|c|c|c|c|c|}
\hline Name & $\begin{array}{c}\mathrm{L} \\
\text { Degrees }\end{array}$ & $\begin{array}{c}\text { B } \\
\text { Degrees }\end{array}$ & $\begin{array}{c}\mathrm{RV} \\
\mathrm{km} \mathrm{s}^{-1}\end{array}$ & $\begin{array}{c}\Delta \mathrm{RV} \\
\mathrm{km} \mathrm{s}^{-1}\end{array}$ & RV Source & $\begin{array}{c}\mathrm{D} \\
\mathrm{Mpc}\end{array}$ & $\begin{array}{l}\Delta \mathrm{D} \\
\mathrm{Mpc}\end{array}$ & D Source & D Method & Absorption & B Mag & K Mag & $\Delta \mathrm{K}$ & Mag & K Mag Sol \\
\hline M95 & 94.09 & -27.08 & 778.0 & 4.0 & NED & 9.33 & 0.39 & 8 & Ceph & 0.120 & 10.53 & 6.67 & & 0.04 & $2 \mathrm{M}$ \\
\hline M96 & 94.29 & -26.41 & 897.0 & 4.0 & NED & 9.86 & 0.27 & 8 & Ceph & 0.109 & 10.11 & 6.32 & & 0.02 & $2 \mathrm{M}$ \\
\hline SextansB & 95.46 & -39.62 & 301.0 & 1.0 & NED & 1.33 & 0.04 & $12,3,34$ & TRGB & 0.137 & 11.85 & $\ldots$ & & $\ldots$ & $\ldots$ \\
\hline M66 & 96.58 & -18.42 & 727.0 & 3.0 & NED & 9.38 & 0.37 & 8 & Ceph & 0.140 & 9.65 & 5.88 & & 0.02 & $2 \mathrm{M}$ \\
\hline DDO187 & 97.83 & 24.35 & 154.0 & 4.0 & NED & 2.50 & 0.20 & 35 & TRGB & 0.105 & 14.38 & $\ldots$ & & $\ldots$ & $\ldots$ \\
\hline GR8 & 102.98 & 4.67 & 214.0 & 3.0 & NED & 2.22 & 0.26 & 36,37 & TRGB & 0.113 & 14.68 & $\ldots$ & & $\ldots$ & $\ldots$ \\
\hline Sextans & 105.39 & -39.23 & 224.0 & 2.0 & NED & 0.10 & 0.00 & 38 & CMD & 0.215 & 12.00 & $\ldots$ & & $\ldots$ & $\ldots$ \\
\hline SextansA & 109.01 & -40.66 & 324.0 & 1.0 & NED & 1.32 & 0.04 & 39 & Ceph,TRGB400 & 0.188 & 11.86 & $\ldots$ & & $\ldots$ & $\ldots$ \\
\hline KKH86 & 116.34 & 15.47 & 283.0 & 3.0 & NED & 2.61 & 0.16 & 3 & TRGB & 0.118 & 16.80 & $\ldots$ & & $\ldots$ & $\ldots$ \\
\hline NGC3109 & 137.96 & -45.10 & 403.0 & 1.0 & NED & 1.32 & 0.03 & $12,3,40,41$ & TRGB,TRGB,Ceph,TRGB & 0.288 & 10.39 & 9.28 & & 0.07 & $2 \mathrm{M}$ \\
\hline Antlia & 139.93 & -44.80 & 351.0 & 15.0 & 42 & 1.32 & 0.10 & 43 & TRGB & 0.342 & 16.19 & $\ldots$ & & $\ldots$ & $\ldots$ \\
\hline NGC3621 & 145.65 & -28.57 & 727.0 & 5.0 & NED & 6.43 & 0.31 & 44,8 & Ceph & 0.346 & 10.18 & 6.60 & & 0.04 & $2 \mathrm{M}$ \\
\hline PGC48111 & 146.23 & 1.64 & 587.0 & 4.0 & NED & 4.61 & 0.40 & 45 & TRGB & 0.295 & 14.96 & $\ldots$ & & $\ldots$ & $\ldots$ \\
\hline KK98-112 & 146.93 & -20.99 & 640.0 & 5.0 & NED & 5.22 & 0.43 & 45 & TRGB & 0.323 & 16.60 & $\ldots$ & 1 & $\ldots$ & $\ldots$ \\
\hline IC4316 & 147.25 & 1.98 & 674.0 & 53.0 & NED & 4.41 & 0.47 & 45 & TRGB & 0.237 & 14.97 & $\ldots$ & 0 & $\ldots$ & $\ldots$ \\
\hline KK98-208 & 147.62 & 1.00 & 381.0 & 0.0 & NED & 4.68 & 0.41 & 45 & TRGB & 0.192 & 14.30 & $\ldots$ & I & $\ldots$ & $\ldots$ \\
\hline M83 & 147.93 & 0.99 & 516.0 & 4.0 & NED & 4.27 & 0.31 & 46 & Ceph & 0.284 & 8.20 & 4.62 & & 0.03 & $2 \mathrm{M}$ \\
\hline KK98-200 & 148.16 & -1.90 & 485.0 & 3.0 & NED & 4.63 & 0.31 & 45 & TRGB & 0.298 & 16.67 & $\ldots$ & & $\ldots$ & $\ldots$ \\
\hline NGC5264 & 148.30 & 1.92 & 478.0 & 3.0 & NED & 4.53 & 0.55 & 45 & TRGB & 0.223 & 12.60 & 10.54 & & 0.05 & $2 \mathrm{M}$ \\
\hline NGC5253 & 149.81 & 1.01 & 404.0 & 4.0 & NED & 3.25 & 0.21 & 8 & Ceph & 0.242 & 10.87 & 8.25 & & 0.04 & $2 \mathrm{M}$ \\
\hline PGC39032 & 152.26 & -17.62 & 613.0 & 10.0 & NED & 3.18 & 0.25 & 45 & TRGB & 0.400 & 15.16 & $\ldots$ & & $\ldots$ & $\ldots$ \\
\hline NGC5102 & 153.42 & -4.07 & 467.0 & 7.0 & NED & 3.40 & 0.39 & 45 & TRGB & 0.237 & 10.35 & 6.92 & & 0.04 & $2 \mathrm{M}$ \\
\hline PGC47171 & 158.39 & -4.42 & 513.0 & 6.0 & NED & 3.73 & 0.43 & 45 & TRGB & 0.466 & 12.90 & $\ldots$ & & $\ldots$ & $\ldots$ \\
\hline NGC5128 & 159.75 & -5.25 & 547.0 & 5.0 & NED & 3.75 & 0.28 & 47 & TRGBK & 0.496 & 7.84 & 3.94 & & 0.02 & $2 \mathrm{M}$ \\
\hline PGC48738 & 159.79 & -1.46 & 541.0 & 4.0 & NED & 3.40 & 0.39 & 45 & TRGB & 0.376 & 13.99 & $\ldots$ & & $\ldots$ & $\ldots$ \\
\hline NGC5408 & 160.59 & 1.90 & 509.0 & 7.0 & NED & 4.81 & 0.38 & 45 & TRGB & 0.298 & 12.20 & 11.39 & & 0.14 & $2 \mathrm{M}$ \\
\hline PGC51659 & 166.95 & 3.84 & 397.0 & 68.0 & NED & 3.58 & 0.33 & 45 & TRGB & 0.561 & 16.50 & $\ldots$ & & $\ldots$ & $\ldots$ \\
\hline MilkyWay & 185.79 & 42.31 & -9.0 & 5.0 & 48 & 0.01 & 0.00 & 49 & various & 0.000 & -5.98 & -9.18 & & 0.50 & 50,51 \\
\hline IC3104 & 195.83 & -17.06 & 430.0 & 5.0 & NED & 2.27 & 0.19 & 3 & TRGB & 1.701 & 13.63 & 13.61 & & 0.35 & $2 \mathrm{M}$ \\
\hline NGC2915 & 197.37 & -26.06 & 468.0 & 5.0 & NED & 3.78 & 0.45 & 4 & TRGB405 & 1.185 & 13.25 & 9.83 & & 0.06 & $2 \mathrm{M}$ \\
\hline PGC20125 & 204.47 & -47.15 & 554.0 & 10.0 & NED & 4.90 & 0.50 & 4 & TRGB405 & 0.513 & 14.20 & $\ldots$ & & $\ldots$ & $\ldots$ \\
\hline Carina & 210.10 & -54.64 & 229.0 & 60.0 & NED & 0.09 & 0.01 & 52 & CMD & 0.271 & 10.80 & $\ldots$ & & $\ldots$ & $\ldots$ \\
\hline LMC & 215.80 & -34.12 & 278.0 & 2.0 & NED & 0.05 & 0.00 & fixed & various & 0.324 & 0.90 & $\ldots$ & & $\ldots$ & $\ldots$ \\
\hline SagDIG & 221.27 & 55.52 & -77.0 & 4.0 & NED & 1.05 & 0.05 & 3,53 & TRGB & 0.522 & 15.50 & $\ldots$ & & $\ldots$ & $\ldots$ \\
\hline
\end{tabular}


Table 1-Continued

\begin{tabular}{|c|c|c|c|c|c|c|c|c|c|c|c|c|c|c|}
\hline Name & $\begin{array}{c}\mathrm{L} \\
\text { Degrees }\end{array}$ & $\begin{array}{c}\text { B } \\
\text { Degrees }\end{array}$ & $\begin{array}{c}\mathrm{RV} \\
\mathrm{km} \mathrm{s}^{-1}\end{array}$ & $\begin{array}{c}\Delta \mathrm{RV} \\
\mathrm{km} \mathrm{s}^{-1}\end{array}$ & RV Source & $\begin{array}{c}\mathrm{D} \\
\mathrm{Mpc}\end{array}$ & $\begin{array}{l}\Delta \mathrm{D} \\
\mathrm{Mpc}\end{array}$ & D Source & D Method & Absorption & B Mag & K Mag & $\Delta \mathrm{K}$ Mag & K Mag Sour \\
\hline SMC & 224.23 & -14.83 & 158.0 & 4.0 & NED & 0.06 & 0.00 & 54 & $\mathrm{~EB}$ & 0.160 & 2.70 & $\ldots$ & $\ldots$ & $\ldots$ \\
\hline PGC19337 & 226.90 & -78.97 & 499.0 & 1.0 & NED & 4.23 & 0.45 & 4 & TRGB405 & 0.337 & 13.69 & $\ldots$ & $\ldots$ & $\ldots$ \\
\hline Tucana & 227.61 & -0.92 & 182.0 & 10.0 & 55 & 0.87 & 0.06 & 56 & TRGB & 0.137 & 15.70 & $\ldots$ & $\ldots$ & $\ldots$ \\
\hline NGC1313 & 227.98 & -28.22 & 475.0 & 3.0 & NED & 4.13 & 0.11 & 12 & TRGB & 0.471 & 9.20 & 7.57 & 0.06 & $2 \mathrm{M}$ \\
\hline NGC6822 & 229.08 & 57.10 & -57.0 & 2.0 & NED & 0.48 & 0.02 & 57,58 & TRGB,RRL & 1.020 & 9.31 & 6.72 & 0.05 & $2 \mathrm{M}$ \\
\hline NGC1705 & 231.83 & -45.54 & 628.0 & 9.0 & NED & 5.11 & 0.61 & 59 & TRGB & 0.035 & 12.77 & 10.53 & 0.06 & $2 \mathrm{M}$ \\
\hline IC5152 & 234.23 & 11.53 & 124.0 & 3.0 & NED & 1.88 & 0.20 & 60,3 & TRGB & 0.106 & 11.06 & 9.28 & 0.03 & $2 \mathrm{M}$ \\
\hline PGC09962 & 235.18 & -25.96 & 513.0 & 9.0 & NED & 4.66 & 0.47 & 4 & TRGB405 & 0.110 & 13.24 & 13.57 & 0.19 & $2 \mathrm{M}$ \\
\hline KK98-54 & 237.66 & -77.50 & 495.0 & 22.0 & NED & 4.99 & 0.60 & 4 & TRGB405 & 0.283 & 15.60 & $\ldots$ & $\ldots$ & $\ldots$ \\
\hline DDO210 & 252.08 & 50.24 & -140.7 & 1.3 & 61 & 0.94 & 0.03 & 62,3 & TRGB & 0.221 & 14.00 & $\ldots$ & $\ldots$ & $\ldots$ \\
\hline Phoenix & 254.29 & -20.86 & -13.0 & 9.0 & 88 & 0.40 & 0.01 & 63,64 & TRGB & 0.067 & 13.07 & $\ldots$ & $\ldots$ & $\ldots$ \\
\hline PGC01641 & 254.37 & -5.27 & 117.0 & 5.0 & NED & 1.92 & 0.09 & 3 & TRGB & 0.024 & 15.60 & $\ldots$ & $\ldots$ & $\ldots$ \\
\hline ESO245-05 & 255.14 & -19.74 & 395.0 & 6.0 & NED & 4.43 & 0.45 & 65 & TRGB405 & 0.069 & 12.70 & $\ldots$ & $\ldots$ & $\ldots$ \\
\hline NGC625 & 257.27 & -17.74 & 396.0 & 1.0 & NED & 3.89 & 0.22 & 66 & TRGB & 0.071 & 11.71 & 9.09 & 0.04 & $2 \mathrm{M}$ \\
\hline UGCA438 & 258.88 & 9.28 & 62.0 & 5.0 & NED & 2.16 & 0.10 & 3,67 & TRGB & 0.064 & 13.86 & $\ldots$ & $\ddot{F} \ldots$ & $\ldots$ \\
\hline NGC300 & 259.81 & -9.50 & 144.0 & 1.0 & NED & 2.02 & 0.07 & 8 & Ceph & 0.055 & 8.95 & 6.38 & 0.06 & $2 \mathrm{M}$ \\
\hline UGCA442 & 260.78 & 6.12 & 267.0 & 8.0 & NED & 4.27 & 0.52 & 65 & TRGB405 & 0.072 & 13.60 & 12.77 & 0.33 & $2 \mathrm{M}$ \\
\hline NGC7793 & 261.30 & 3.12 & 230.0 & 2.0 & NED & 3.91 & 0.41 & 65 & TRGB405 & 0.084 & 9.98 & 6.86 & 0.06 & $2 \mathrm{M}$ \\
\hline Sculptor & 263.98 & -9.68 & 110.0 & 1.0 & NED & 0.08 & 0.00 & 68 & RRL & 0.077 & 10.05 & $\ldots$ & $\ldots$ & $\ldots$ \\
\hline Fornax & 265.37 & -30.27 & 53.0 & 9.0 & NED & 0.14 & 0.01 & 69 & RRL & 0.087 & 9.28 & $\ldots$ & $\ldots$ & $\ldots$ \\
\hline NGC253 & 271.57 & -5.01 & 241.0 & 2.0 & NED & 3.94 & 0.37 & 65 & TRGB405 & 0.081 & 8.04 & 3.77 & 0.02 & $2 \mathrm{M}$ \\
\hline IC1574 & 274.23 & -3.21 & 361.0 & 7.0 & NED & 4.92 & 0.58 & 65 & TRGB405 & 0.065 & 15.09 & $\ldots$ & $\ldots$ & $\ldots$ \\
\hline DDO6 & 275.84 & -4.40 & 301.0 & 5.0 & NED & 3.34 & 0.24 & 65 & TRGB405 & 0.073 & 15.19 & $\cdots$ & $\ldots$ & $\cdots$ \\
\hline WLM & 277.81 & 8.09 & -116.0 & 2.0 & NED & 0.89 & 0.04 & 70 & TRGB & 0.160 & 11.03 & $\ldots$ & $\ldots$ & $\ldots$ \\
\hline IC1613 & 299.15 & -1.78 & -234.0 & 1.0 & NED & 0.73 & 0.02 & $8,71,72$ & Ceph,various,TRGB & 0.108 & 9.88 & 12.77 & 0.11 & $2 \mathrm{M}$ \\
\hline DDO216 & 305.83 & 24.30 & -183.3 & 1.3 & 61 & 0.76 & 0.10 & 73 & CMD & 0.284 & 13.21 & $\ldots$ & $\ldots$ & $\ldots$ \\
\hline UGC685 & 313.34 & 1.61 & 157.0 & 1.0 & NED & 4.79 & 0.30 & 31 & TRGB & 0.246 & 14.23 & $\ldots$ & $\ldots$ & $\ldots$ \\
\hline AndVI & 317.31 & 20.56 & -354.0 & 3.0 & NED & 0.77 & 0.03 & 74,75 & TRGB,Cep & 0.276 & 14.17 & $\ldots$ & $\ldots$ & $\ldots$ \\
\hline LGS3 & 318.13 & 3.80 & -277.0 & 5.0 & NED & 0.62 & 0.02 & 76 & TRGB,HB,RC & 0.177 & 14.20 & $\ldots$ & $\ldots$ & $\ldots$ \\
\hline KK98-16 & 327.50 & -5.41 & 205.0 & 10.0 & 20 & 4.74 & 0.53 & 4 & TRGB405 & 0.301 & 16.30 & $\ldots$ & $\ldots$ & $\ldots$ \\
\hline M33 & 328.47 & -0.09 & -179.0 & 3.0 & NED & 0.80 & 0.02 & $8,77,78$ & Ceph,TRGBClump,Ceph & 0.181 & 6.27 & 4.10 & 0.04 & $2 \mathrm{M}$ \\
\hline KK98-17 & 328.71 & -6.08 & 155.0 & 10.0 & 20 & 4.72 & 0.41 & 4 & TRGB405 & 0.239 & 17.20 & $\ldots$ & $\ldots$ & $\ldots$ \\
\hline AndII & 330.01 & 4.23 & -188.0 & 3.0 & NED & 0.66 & 0.01 & $79,80,78$ & RRL,TRGB & 0.269 & 13.50 & $\ldots$ & $\ldots$ & $\ldots$ \\
\hline AndIII & 331.13 & 13.10 & -351.0 & 9.0 & NED & 0.76 & 0.07 & 81 & TRGB & 0.244 & 15.00 & $\ldots$ & $\ldots$ & $\ldots$ \\
\hline
\end{tabular}


Table 1-Continued

\begin{tabular}{|c|c|c|c|c|c|c|c|c|c|c|c|c|c|c|}
\hline Name & $\begin{array}{c}\mathrm{L} \\
\text { Degrees }\end{array}$ & $\begin{array}{c}\text { B } \\
\text { Degrees }\end{array}$ & $\begin{array}{c}\mathrm{RV} \\
\mathrm{km} \mathrm{s}^{-1}\end{array}$ & $\begin{array}{c}\Delta \mathrm{RV} \\
\mathrm{km} \mathrm{s}^{-1}\end{array}$ & RV Source & $\begin{array}{c}\mathrm{D} \\
\mathrm{Mpc}\end{array}$ & $\begin{array}{l}\Delta \mathrm{D} \\
\mathrm{Mpc}\end{array}$ & D Source & D Method & Absorption & B Mag & K Mag & $\Delta \mathrm{K}$ Mag & K Mag Source \\
\hline NGC404 & 331.85 & 6.25 & -48.0 & 9.0 & NED & 3.20 & 0.21 & 82,3 & TRGB & 0.253 & 11.21 & 8.57 & 0.03 & 89 \\
\hline ККH98 & 332.35 & 23.17 & -136.0 & 3.0 & NED & 2.45 & 0.12 & 3 & TRGB & 0.532 & 16.70 & $\ldots$ & $\ldots$ & $\ldots$ \\
\hline AndI & 333.05 & 11.41 & -368.0 & 11.0 & NED & 0.76 & 0.03 & 80,78 & TRGB & 0.234 & 13.60 & $\ldots$ & $\ldots$ & $\ldots$ \\
\hline NGC925 & 335.45 & -9.47 & 553.0 & 3.0 & NED & 9.12 & 0.17 & 8 & Ceph & 0.326 & 10.69 & 8.74 & 0.03 & $2 \mathrm{M}$ \\
\hline M31 & 336.19 & 12.55 & -300.0 & 4.0 & NED & 0.75 & 0.02 & 8 & Ceph & 0.268 & 4.36 & 0.98 & 0.02 & $2 \mathrm{M}$ \\
\hline NGC205 & 336.54 & 13.06 & -241.0 & 3.0 & NED & 0.72 & 0.07 & 83 & TRGB & 0.268 & 8.92 & 5.59 & 0.05 & $2 \mathrm{M}$ \\
\hline KKH18 & 339.26 & -15.93 & 216.0 & 3.0 & NED & 4.43 & 0.49 & 4 & TRGB405 & 0.860 & 16.70 & $\ldots$ & $\ldots$ & $\ldots$ \\
\hline NGC185 & 343.27 & 14.30 & -202.0 & 3.0 & NED & 0.62 & 0.06 & 84 & TRGB & 0.787 & 10.10 & 6.56 & 0.05 & $2 \mathrm{M}$ \\
\hline NGC147 & 343.32 & 15.27 & -193.0 & 3.0 & NED & 0.76 & 0.10 & 85 & RGB/HB & 0.747 & 10.47 & 7.20 & 0.06 & $2 \mathrm{M}$ \\
\hline KKH96 & 345.65 & 26.05 & -307.0 & 5.0 & 1 & 0.79 & 0.04 & 1 & TRGB405 & 0.846 & 13.60 & $\ldots$ & $\ldots$ & $\ldots$ \\
\hline KKH5 & 347.21 & 10.31 & 61.0 & 2.0 & NED & 4.27 & 0.33 & 4 & TRGB405 & 1.218 & 17.10 & $\ldots$ & $\ldots$ & $\ldots$ \\
\hline $\mathrm{IC} 10$ & 354.43 & 17.88 & -348.0 & 1.0 & NED & 0.66 & 0.08 & 86 & Ceph,TRGB & 6.588 & 11.80 & 6.01 & 0.03 & $2 \mathrm{M}$ \\
\hline MaffeiI & 359.29 & 1.44 & 66.4 & 5.0 & 87 & 3.01 & 0.30 & 87 & various & 6.120 & 13.47 & 4.68 & 0.02 & $2 \mathrm{M}$ \\
\hline
\end{tabular}

巨

References. - (1) Karachentsev et al. (2003d); (2) Saha et al. (2002); (3) Karachentsev et al. (2002c); (4) Karachentsev et al. (2003b); (5) Drozdovsky et al. (2001); (6) Karachentsev et al. (2002a); (7) Davidge (2003); (8) Freedman et al. (2001); (9) Hoessel et al. (1998a); (10) Izotov \& Thuan (2002); (11) Dolphin et al. (2001b); (12) Méndez et al. (2002); (13) Lynds et al. (1998); (14) Tully et al. (1981); (15) Sakai \& Madore (1999); (16) Karachentsev et al. (2000b); (17) Sakai \& Madore (2001); (18) Bonanos et al. (2004); (19) Sharina et al. (2001); (20) Huchtmeier et al. (2000a); (21) Bellazzini et al. (2002); (22) Huchtmeier et al. (2000b); (23) Karachentsev et al. (2001a); (24) Karachentsev et al. (2003a); (25) Stetson et al. (1998); (26) Herrnstein et al. (1999); (27) Tolstoy et al. (1998a); (28) Dolphin et al. (2002); (29) Aparicio \& Tikhonov (2000); (30) Crone et al. (2000); (31) Maíz-Apellániz et al. (2002); (32) Drozdovsky et al. (2002); (33) Thim et al. (2004); (34) Sakai, Madore \& Freedman (1997); (35) Aparicio et al. (2000); (36) Dohm-Palmer et al. (1998); (37) Tolstoy (1995); (38) Lee et al. (2003); (39) Dolphin et al. (2003); (40) Musella et al. (1997); (41) Hoessel et al. (1998b); (42) Tolstoy \& Irwin (2000); (43) Aparicio et al. (1997); (44) Rawson et al. (1997); (45) Karachentsev et al. (2002b); (46) Thim et al. (2003); (47) Rejkuba (2004); (48) Mihalas \& Binney (1984); (49) Kerr \& Lynden-Bell (1986); (50) Suntzeff et al. (1992); (51) Kent et al. (1991); (52) Mighell (1997); (53) Karachentsev et al. (1999a); (54) Harries et al. (2003); (55) Tolstoy et al. (2004); (56) Saviane et al. (1996); (57) Wyder (2003); (58) Clementini et al. (2003); (59) Tosi et al. (2001); (60) Zijlstra \& Minniti (1999); (61) Young et al. (2003); (62) Lee et al. (1999); (63) Martínez-Delgado et al. (1999); (64) Held et al. (1999); (65) Karachentsev et al. (2003c); (66) Cannon et al. (2003); (67) Lee \& Byun (1999); (68) Kunkel \& Demers (1977); (69) Mackey \& Gilmore (2003); (70) Minniti \& Zijlstra (1997); (71) Dolphin et al. (2001a); (72) Cole et al. (1999); (73) Gallagher et al. (1998); (74) Hopp et al. (1999); (75) Pritzl et al. (2002); (76) Miller et al. (2001); (77) Lee et al. (2002); (78) McConnachie et al. (2004); (79) Pritzl et al. (2004); (80) DaCosta et al. (200); (81) Armandroff et al. (1993); (82) Tikhonov et al. (2003); (83) Mould et al. (1984); (84) Lee, Freedman \& Madore (1993); (85) Han et al. (1997); (86) Sakai, Madore \& Freedman (1999); (87) Fingerhut et al. (2003); (88) Irwin \& Tolstoy (2002); (89) Frogel et al. (1978)

Note. - Observational data on Local Volume galaxies, derived from the literature. NED is the NASA Extragalactic Database (http://nedwww.ipac.caltech.edu/index.html); 2M, the 2MASS survey, data extracted through NED. TRGB, tip of the Red Giant Branch (sometimes using the SDSS filters or $K$, and noted as such); HB, horizonal branch; CMD, color-magnitude diagram; RRL, RR Lyraes; Ceph, Cepheids; Clump or RC, red clump; EB, eclipsing binaries; Geo, a Geometric method using masers. A lack of available data is 
denoted by “...”. 
Table 2. Additional Local Volume Data

\begin{tabular}{|c|c|c|c|c|c|c|c|c|c|c|}
\hline Name & $\begin{array}{c}\mathrm{L} \\
\text { Degrees }\end{array}$ & $\begin{array}{c}\text { B } \\
\text { Degrees }\end{array}$ & $\begin{array}{c}\mathrm{RV} \\
\mathrm{km} \mathrm{s}^{-1}\end{array}$ & $\begin{array}{c}\Delta \mathrm{RV} \\
\mathrm{km} \mathrm{s}^{-1}\end{array}$ & RV Source & $\begin{array}{c}\mathrm{D} \\
\mathrm{Mpc}\end{array}$ & $\begin{array}{l}\Delta \mathrm{D} \\
\mathrm{Mpc}\end{array}$ & D Source & D Method & Absorption \\
\hline NGC6946 & 10.03 & 42.00 & 48.0 & 2.0 & NED & 6.80 & 1.40 & 1 & stars & 1.475 \\
\hline NGC1569 & 11.91 & -4.92 & -104.0 & 4.0 & NED & 1.95 & 0.20 & 2 & TRGB* & 3.020 \\
\hline NGC2683 & 55.87 & -33.42 & 411.0 & 1.0 & NED & 9.20 & 1.80 & 3 & stars & 0.142 \\
\hline NGC5585 & 60.40 & 24.67 & 305.0 & 3.0 & NED & 8.70 & 1.70 & 3 & stars & 0.067 \\
\hline NGC5195 & 71.09 & 17.35 & 465.0 & 10.0 & NED & 7.66 & 0.95 & 4 & SBF & 0.155 \\
\hline NGC5194 & 71.17 & 17.32 & 463.0 & 3.0 & NED & 7.66 & 0.95 & 4 & SBF & 0.150 \\
\hline NGC2903 & 73.53 & -36.44 & 556.0 & 1.0 & NED & 8.90 & 1.80 & 3 & stars & 0.134 \\
\hline NGC3344 & 81.17 & -21.09 & 586.0 & 4.0 & NED & 7.70 & 1.20 & 5 & rv & 0.143 \\
\hline NGC4826 & 95.61 & 6.13 & 408.0 & 4.0 & NED & 7.48 & 0.69 & 4 & SBF & 0.178 \\
\hline NGC3115 & 112.40 & -42.86 & 720.0 & 5.0 & NED & 9.68 & 0.40 & 4 & SBF & 0.205 \\
\hline NGC2784 & 136.74 & -56.74 & 691.0 & 35.0 & NED & 9.82 & 1.70 & 4 & SBF & 0.925 \\
\hline NGC5068 & 138.28 & -0.21 & 673.0 & 5.0 & NED & 6.90 & 1.20 & 5 & $\mathrm{rv}$ & 0.439 \\
\hline NGC4945 & 165.18 & -10.21 & 560.0 & 5.0 & NED & 4.10 & 1.20 & 5 & $\mathrm{rv}$ & 0.762 \\
\hline ESO274G01 & 171.62 & 10.29 & 522.0 & 5.0 & NED & 4.70 & 1.20 & 5 & $\mathrm{rv}$ & 1.108 \\
\hline Circinus & 183.14 & -6.42 & 449.0 & 10.0 & NED & 2.70 & 1.20 & 5 & $\mathrm{rv}$ & 2.000 \\
\hline IC5052 & 216.46 & 2.59 & 598.0 & 5.0 & NED & 6.60 & 1.20 & 5 & $\mathrm{rv}$ & 0.219 \\
\hline NGC55 & 256.25 & -2.36 & 129.0 & 3.0 & NED & 2.10 & 0.50 & 6 & various & 0.057 \\
\hline NGC247 & 275.92 & -3.73 & 160.0 & 2.0 & NED & 3.21 & 0.50 & 6 & various & 0.078 \\
\hline NGC628 & 314.53 & -5.39 & 657.0 & 1.0 & NED & 7.32 & 1.50 & 7 & stars & 0.301 \\
\hline NGC891 & 342.97 & -4.75 & 528.0 & 4.0 & NED & 8.36 & 0.54 & 4 & SBF & 0.280 \\
\hline Maffei2 & 359.58 & 0.83 & -17.0 & 5.0 & NED & 2.80 & 1.20 & 8 & $\mathrm{TF}$ & 7.190 \\
\hline
\end{tabular}

B Mag K Mag

$\Delta \mathrm{K}$ Mag

K Mag

$\begin{array}{rlll}9.61 & 5.37 & 0.03 & 2 \mathrm{M} \\ 11.86 & 7.86 & 0.02 & 2 \mathrm{M} \\ 10.60 & 6.33 & 0.02 & 2 \mathrm{M} \\ 11.20 & 9.50 & 0.05 & 2 \mathrm{M} \\ 10.45 & 6.25 & 0.03 & 2 \mathrm{M} \\ 8.96 & 5.50 & 0.03 & 2 \mathrm{M} \\ 9.68 & 6.04 & 0.02 & 2 \mathrm{M} \\ 10.45 & 7.44 & 0.04 & 2 \mathrm{M} \\ 9.36 & 5.33 & 0.02 & 2 \mathrm{M} \\ 9.87 & 5.88 & 0.02 & 2 \mathrm{M} \\ 11.30 & 6.32 & 0.02 & 2 \mathrm{M} \\ 10.52 & 7.55 & 0.05 & 2 \mathrm{M} \\ 9.30 & 4.48 & 0.02 & 2 \mathrm{M} \\ 11.70 & 8.36 । & 0.05 & 2 \mathrm{M} \\ 9.61 & 4.98 & 0.03 & 2 \mathrm{M} \\ 11.16 & 8.88 & 0.06 & 2 \mathrm{M} \\ 8.84 & 6.25 & 0.05 & 2 \mathrm{M} \\ 9.86 & 7.43 & 0.06 & 2 \mathrm{M} \\ 9.95 & 6.84 & 0.05 & 2 \mathrm{M} \\ 10.81 & 5.94 & 0.02 & 2 \mathrm{M} \\ 14.27 & 5.21 & 0.03 & 2 \mathrm{M}\end{array}$

References. - (1), Karachentsev et al. (2000a); (2), Makarova \& Karachentsev (2003); (3), Drozdovsky \& Karachentsev (2000); (4), Tonry et al. (2001); (5), this paper; (6), Puche \& Carignan (1988) (modified, see text); (7), Sharina et al. (1996); (8), Karachentsev et al. (2004)

Note. - Observational data on Local Volume galaxies whose distance is not as well known, but which are among the brightest in the Volume. TRGB*, (uncertain) tip of the Red Giant Branch; stars, brightest stars; SBF, surface-brightness fluctuations rv, radial-velocity (assuming zero peculiar velocity and applying the kinematic model derived in this paper); TF, Tully-Fisher relation. The absorption for Circinus was taken from Freeman et al. (1977), all others from Schlegel et al. (1998) by way of NED. 


\section{Analysis}

Before we can begin to look at peculiar velocities we need some way of separating them from background motion.

\subsection{Peculiar Motion}

The motion of a particle $i$ under the gravitational influence of a mass-field $\rho\left(\mathbf{r}^{\prime}\right)$ is governed by the equation

$$
\frac{d \mathbf{v}_{i}}{d t}=G \int \frac{\rho\left(\mathbf{r}^{\prime}-\mathbf{r}_{i}\right)}{\left|\mathbf{r}^{\prime}-\mathbf{r}_{i}\right|^{3}} d \mathbf{r}^{\prime}
$$

where $\mathbf{r}_{i}$ is the location of the particle. In principle, the (three-dimensional) integral is taken over all space occupied by matter; in practice, we restrict ourselves to a limited region, and take any part of the universe outside to be isotropic, and thus to have no effect.

If we separate $\rho$ into a smooth background density $\rho_{b}$ and a fluctuating part, and in addition assume that the fluctuations can be adequately modelled by point masses, the equation of motion becomes

$$
\begin{aligned}
\frac{d \mathbf{v}_{i}}{d t} & =G \int \frac{\rho_{b}\left(\mathbf{r}^{\prime}-\mathbf{r}_{i}\right)}{\left|\mathbf{r}^{\prime}-\mathbf{r}_{i}\right|^{3}}+G \sum_{j \neq i} \frac{m_{j}\left(\mathbf{r}^{\prime}-\mathbf{r}_{i}\right)}{\left|\mathbf{r}^{\prime}-\mathbf{r}_{i}\right|^{3}} \\
& =\frac{d \mathbf{v}_{b}}{d t}+\frac{d \mathbf{v}_{f}}{d t}
\end{aligned}
$$

so the acceleration splits into that induced by a smooth background $(b)$ and that caused by fluctuations $(f)$. When integrated over time the former will be a smooth Hubble flow, the latter the peculiar velocity. Note that if attention is restricted to a part of the universe, the background may have a different value than another part; so that, for instance, the "Hubble constant" within $10 \mathrm{Mpc}$ is not necessarily that of the universe as a whole.

\subsection{Kinematic Background Solutions}

In practice, we must calculate the background solution from data at hand. Smooth motion is modelled as a contant average velocity $\mathbf{v}$ plus an expansion, which might be anisotropic, represented by a tensor $\mathbf{H}$. (Strictly speaking, an anisotropic expansion reflects peculiar velocity, as one might expect from an external tidal force; this will be treated below. It is convenient, however, to calculate it as part of the background.) We take as a measure 
of goodness of fit of the model to the data the average square of the difference between the predicted radial velocity and the observed radial velocity,

$$
\sigma^{2}=\frac{1}{N} \sum_{i} \sigma_{i}^{2}=\frac{1}{N} \sum_{i}\left(v_{\mathrm{obs}}-\left(\mathbf{v}_{0} \cdot \hat{\mathbf{r}}+\hat{\mathbf{r}} \cdot \mathbf{H} \cdot \mathbf{r}\right)\right)^{2}
$$

The parameters of the model, $\mathbf{v}_{0}$ and the six independent components of $\mathbf{H}$, are found analytically by taking the derivative of $\sigma^{2}$ with respect to each. Then setting the resulting expressions equal to zero gives nine linear equations to be solved for the nine unknowns, a straightforward if tedious calculation. An isotropic solution is determined similarly, using four equations in four unknowns. (A true maximum-likelihood solution could be constructed by weighting the various data points with their observational errors. As will be seen, in this case the errors are a minor contributor to scatter about the solutions, and the additional complication of weighting would not make any significant difference in the solutions.)

In determining these background solutions, as well as in any calculations depending on peculiar velocities, only the 149-galaxy data set is used.

It is worth emphasizing that the background solutions are kinematic, and thus independent of any specific cosmology. That is, they do not assume a value for $\rho_{b}$, or $\Lambda$, or anything else. The peculiar velocites derived from them are thus not affected by variations in such parameters.

It is important to know just how certain the derived background parameters are. For this the error-tensor method of Whiting (2003) is used; details are set out in that paper. Briefly, this uses an analytical formula to measure how much a certain parameter can be changed before the spread of data points about the solution becomes significantly greater (using the F-ratio test). The numbers quoted correspond to a $68 \%$ chance of the modified model being significantly worse, and are thus comparable to a standard one-sigma error bar. There are two obvious ways this approach can fail: first, the distribution of points around the solution can be non-Gaussian. In some situations in this paper, for instance the distribution of errors in synthetic gravity, a Gaussian is very wrong; but not in those in which I have employed the error tensor.

Second, a change in two or more parameters together may cause less increase in dispersion than one alone. This way both are more uncertain than each considered individually might be. This is shown by significant off-diagonal terms in the error tensor; for calculations herein, they were not important.

The model parameters, along with similar results from other efforts, are shown in Table $(3)$. 
Table 3. Background Solutions

\begin{tabular}{|c|c|c|c|}
\hline Quantity & Magnitude & $\mathrm{L}$ & $\mathrm{B}$ \\
\hline & $\mathrm{km} \mathrm{s}^{-1}$ or $\mathrm{km} \mathrm{s}^{-1} \mathrm{Mpc}^{-1}$ & Degrees & Degrees \\
\hline \multicolumn{4}{|l|}{ Isotropic } \\
\hline V & $336 \pm 52$ & $11 \pm 8$ & $40 \pm 8$ \\
\hline $\mathrm{H}$ & $66 \pm 6$ & & \\
\hline$\sigma$ & 79 & & \\
\hline \multicolumn{4}{|l|}{ Anisotropic } \\
\hline $\mathrm{V}$ & 352 & 14 & 48 \\
\hline$H_{x x}$ & $86 \pm 18$ & 119 & 15 \\
\hline$H_{y y}$ & $53 \pm 8$ & 23 & 20 \\
\hline$H_{z z}$ & $39 \pm 20$ & 242 & 65 \\
\hline$\sigma$ & 72 & & \\
\hline \multicolumn{4}{|c|}{ (1) Isotropic (98) } \\
\hline $\mathrm{V}$ & $290 \pm 90$ & 10 & 45 \\
\hline $\mathrm{H}$ & 64 & & \\
\hline$\sigma$ & 118 & & \\
\hline \multicolumn{4}{|c|}{ (1) Anisotropic (98) } \\
\hline $\mathrm{V}$ & $310 \pm 90$ & 350 & 49 \\
\hline$H_{x x}$ & $83 \pm 15$ & 127 & 3 \\
\hline$H_{y y}$ & $51 \pm 14$ & 34 & 46 \\
\hline$H_{z z}$ & $32 \pm 16$ & 320 & 44 \\
\hline$\sigma$ & 103 & & \\
\hline \multicolumn{4}{|c|}{ (1) Isotropic (35) } \\
\hline $\mathrm{V}$ & $350 \pm 80$ & 355 & 44 \\
\hline $\mathrm{H}$ & 70 & & \\
\hline$\sigma$ & 89 & & \\
\hline \multicolumn{4}{|c|}{ (1) Anisotropic (35) } \\
\hline $\mathrm{V}$ & $330 \pm 90$ & 350 & 49 \\
\hline$H_{x x}$ & $138 \pm 61$ & 346 & -65 \\
\hline$H_{y y}$ & $84 \pm 18$ & 104 & -12 \\
\hline$H_{z z}$ & $35 \pm 16$ & 19 & 21 \\
\hline$\sigma$ & 77 & & \\
\hline \multicolumn{4}{|l|}{$(2)$} \\
\hline $\mathrm{V}$ & 334 & 22 & 29 \\
\hline \multicolumn{4}{|l|}{ (3) Anisotropic } \\
\hline $\mathrm{V}$ & 325 & 11 & 41 \\
\hline$H_{x x}$ & $82 \pm 3$ & 132 & 0 \\
\hline$H_{y y}$ & $62 \pm 3$ & 42 & 0 \\
\hline
\end{tabular}


Table 3-Continued

\begin{tabular}{lccc}
\hline \hline Quantity & Magnitude & L & B \\
\hline$H_{z z}$ & $48 \pm 5$ & 0 & 90 \\
\hline
\end{tabular}

References. - (1), Whiting (2003); (2), Yahil et al. (1977); (3), Karachentsev \& Makarov (2001).

Note. - Parameters of the local galaxy flow derived in this and other papers: V, the solar reflex velocity (reciprocal of the average flow with respect to the Sun); $\mathrm{H}$, effective (isotropic) Hubble constant; $H_{x x}$ etc., eigenvectors of the anisotropic Hubble flow. Listed uncertainties in Hubble tensor components in Karachentsev \& Makarov (2001) were calculated in a different way from the other papers. The V of Yahil et al. (1977) was determined using only Local Group galaxies. The solutions from Whiting (2003) are for the larger (98-galaxy) sample of less reliable data as well as for a smaller (35-galaxy) sample of better data. 
The first thing to point out is the velocity dispersion around the models. Of the 79 and $72 \mathrm{~km} \mathrm{~s}^{-1}$, the listed observational errors contribute $24 \mathrm{~km} \mathrm{~s}^{-1}$, leaving 75 and $66 \mathrm{~km} \mathrm{~s}^{-1}$ of actual motion. It is worth emphasizing that these figures are signal, not noise; motions to be explained, not random errors to be minimized or in which some "real" object or correlation is hiding.

Second, the solar reflex velocity (the reciprocal of the average velocity of the galaxies within the Volume) is fairly well-determined, to within less than ten degrees in direction and ten or twenty $\mathrm{km} \mathrm{s}^{-1}$. (The latter is much better than the assigned error in the latest isotropic calculation; this may be because the various calculations reuse many of the same data.)

The magnitudes of the components of the Hubble tensor also seem well-determined. However, the directions vary greatly, so the tensor itself cannot be said to agree well among the various calculations. Note the listed (68\%, one-sigma) uncertainties of the (present paper) eigenvalues: each overlaps the next. If $90 \%$ uncertainties are used we have $86 \pm 31$, $53 \pm 14$ and $40 \pm 34 \mathrm{~km} \mathrm{~s}^{-1} \mathrm{Mpc}^{-1}$, and all three overlap. The best that can be said is that some anisotropy may have been detected at a one-sigma level. (The errors listed for Karachentsev \& Makarov (2001) are formal errors, and appear to be rather optimistic about the real uncertainty in anisotropic flow.)

How much anisotropy should we expect? Klypin et al. (2003) performed N-body simulations constrained to give the same overall structure as found in the local universe (extending beyond the Local Volume, but with good resolution within it). In their analogue of the Supergalactic Plane most of the peculiar velocities (of something under $100 \mathrm{~km} \mathrm{~s}^{-1}$ ) were perpendicular to that feature; in other words, the major component of peculiar velocities reflected infall into the Plane. In our case, at most 7 or $8 \mathrm{~km} \mathrm{~s}^{-1}$ (the difference between isotropic and anisotropic solutions) out of $75 \mathrm{~km} \mathrm{~s}^{-1}$ can be attributed to SGP infall, about one-tenth.

This is an important result. Whether from dynamical or kinematical arguments, we expect to see a great deal of infall into the largest structure in the Volume, and it's not there.

With the failure of the tensor solution to show much beyond the isotropic solution, we will take the latter as the basis for further calculations. Table (4) shows the absolute magnitudes (in $B$ and $K$ ), with uncertainties, and the peculiar radial velocities for each of the 170 galaxies in the total data set, based on that background. 
Table 4. Derived Local Volume Data

\begin{tabular}{|c|c|c|c|c|c|}
\hline Name & $M_{B}$ & $\Delta M_{B}$ & $M_{K}$ & $\Delta M_{K}$ & Peculiar RV \\
\hline NGC6946 & -21.03 & 0.60 & -23.79 & 0.45 & -75 \\
\hline KK98-35 & -12.64 & 0.46 & -10.00 & 0.22 & -30 \\
\hline IC342 & -20.89 & 0.44 & -23.02 & 0.18 & 59 \\
\hline NGC1569 & -17.61 & 0.46 & -18.59 & 0.22 & -12 \\
\hline UGCA105 & -14.94 & 0.46 & -10.00 & 0.22 & 100 \\
\hline KK98-44 & -12.45 & 0.40 & -10.00 & 0.00 & 75 \\
\hline NGC1560 & -16.34 & 0.46 & -18.76 & 0.24 & -28 \\
\hline KK98-41 & -14.08 & 0.48 & -10.00 & 0.26 & -67 \\
\hline KKH34 & -12.29 & 0.43 & -10.00 & 0.17 & 23 \\
\hline NGC6789 & -14.34 & 0.46 & -15.56 & 0.24 & -77 \\
\hline NGC2366 & -16.25 & 0.49 & -16.90 & 0.31 & 70 \\
\hline NGC2403 & -18.74 & 0.44 & -21.31 & 0.18 & 85 \\
\hline NGC6503 & -17.84 & 0.51 & -21.31 & 0.32 & -11 \\
\hline DDO50 & -16.64 & 0.41 & -18.80 & 0.11 & 117 \\
\hline KDG52 & -11.34 & 0.43 & -10.00 & 0.16 & 58 \\
\hline UGC4483 & -12.61 & 0.41 & -10.00 & 0.09 & 111 \\
\hline DDO53 & -13.44 & 0.43 & -10.00 & 0.15 & -61 \\
\hline UGC3755 & -14.82 & 0.42 & -10.00 & 0.11 & -133 \\
\hline VIIZw403 & -13.88 & 0.40 & -15.62 & 0.11 & -176 \\
\hline HoI & -15.13 & 0.48 & -10.00 & 0.26 & 64 \\
\hline M82 & -19.33 & 0.45 & -23.29 & 0.21 & 113 \\
\hline BK3N & -11.45 & 0.42 & -10.00 & 0.13 & -160 \\
\hline M81 & -20.21 & 0.41 & -23.92 & 0.08 & -105 \\
\hline NGC2976 & -17.24 & 0.46 & -20.23 & 0.23 & -74 \\
\hline KK98-81 & -12.49 & 0.43 & -10.00 & 0.15 & -211 \\
\hline NGC3077 & -17.61 & 0.45 & -20.63 & 0.21 & -78 \\
\hline Garland & -10.00 & 0.47 & -10.00 & 0.24 & -40 \\
\hline DDO82 & -14.73 & 0.46 & -10.00 & 0.22 & 88 \\
\hline KK98-85 & -10.08 & 0.43 & -10.00 & 0.15 & -105 \\
\hline DDO71 & -10.13 & 0.43 & -10.00 & 0.15 & -206 \\
\hline IC2574 & -17.38 & 0.46 & -17.30 & 0.25 & -47 \\
\hline
\end{tabular}


Table 4-Continued

\begin{tabular}{lccccl}
\hline \hline \multicolumn{1}{c}{ Name } & $M_{B}$ & $\Delta M_{B}$ & $M_{K}$ & $\Delta M_{K}$ & Peculiar RV \\
\hline Draco & -13.62 & 0.40 & -10.00 & 0.03 & -85 \\
KDG73 & -13.09 & 0.42 & -10.00 & 0.13 & -216 \\
DDO78 & -12.15 & 0.43 & -10.00 & 0.15 & -34 \\
DDO47 & -15.12 & 0.47 & -10.00 & 0.25 & -164 \\
KK98-65 & -12.81 & 0.44 & -10.00 & 0.18 & 13 \\
NGC4236 & -18.25 & 0.46 & -19.23 & 0.22 & -115 \\
UMi & -7.64 & 0.42 & -10.00 & 0.11 & -34 \\
DDO165 & -15.60 & 0.44 & -10.00 & 0.19 & -82 \\
UGC4115 & -13.62 & 0.46 & -10.00 & 0.23 & -139 \\
NGC2683 & -19.36 & 0.58 & -23.49 & 0.43 & -224 \\
KKR25 & -9.38 & 0.42 & -10.00 & 0.14 & -41 \\
NGC5204 & -16.66 & 0.47 & -18.83 & 0.25 & 45 \\
NGC3738 & -16.37 & 0.47 & -10.00 & 0.24 & 2 \\
NGC5585 & -18.56 & 0.58 & -20.20 & 0.43 & -100 \\
UGC8508 & -12.71 & 0.42 & -10.00 & 0.13 & 28 \\
M101 & -20.90 & 0.41 & -23.66 & 0.11 & -60 \\
UGC7298 & -13.22 & 0.43 & -10.00 & 0.17 & -11 \\
UGC6541 & -10.00 & 0.48 & -10.00 & 0.26 & 58 \\
NGC3741 & -13.22 & 0.46 & -10.00 & 0.24 & 73 \\
M106 & -20.34 & 0.41 & -23.91 & 0.08 & 26 \\
LeoA & -11.52 & 0.44 & -10.00 & 0.18 & -84 \\
KK98-109 & -10.85 & 0.43 & -10.00 & 0.16 & -34 \\
NGC5195 & -19.13 & 0.48 & -23.17 & 0.27 & 62 \\
NGC5194 & -20.61 & 0.48 & -23.93 & 0.27 & 59 \\
DDO167 & -11.15 & 0.47 & -10.00 & 0.24 & -25 \\
DDO168 & -15.55 & 0.47 & -10.00 & 0.25 & -7 \\
NGC4449 & -18.21 & 0.48 & -20.87 & 0.26 & -15 \\
DDO125 & -14.25 & 0.41 & -10.00 & 0.10 & 82 \\
NGC2903 & -20.20 & 0.59 & -23.71 & 0.44 & -133 \\
DDO190 & -14.08 & 0.42 & -10.00 & 0.12 & 80 \\
DDO99 & -13.82 & 0.44 & -10.00 & 0.17 & 80 \\
& & & & &
\end{tabular}


Table 4-Continued

\begin{tabular}{lrrrrl}
\hline \hline \multicolumn{1}{c}{ Name } & $M_{B}$ & $\Delta M_{B}$ & $M_{K}$ & $\Delta M_{K}$ & Peculiar RV \\
\hline M94 & -19.43 & 0.49 & -23.24 & 0.28 & 51 \\
NGC4244 & -17.47 & 0.46 & -20.54 & 0.23 & -36 \\
UGCA290 & -10.00 & 0.42 & -10.00 & 0.12 & 41 \\
DDO181 & -12.72 & 0.45 & -10.00 & 0.21 & 77 \\
IC3687 & -14.68 & 0.46 & -10.00 & 0.23 & 85 \\
DDO126 & -14.30 & 0.47 & -10.00 & 0.25 & -85 \\
NGC4214 & -17.11 & 0.42 & -19.34 & 0.14 & 113 \\
DDO113 & -11.97 & 0.41 & -10.00 & 0.11 & 102 \\
IC4182 & -15.34 & 0.40 & -10.00 & 0.06 & 62 \\
UGC7605 & -13.30 & 0.48 & -10.00 & 0.26 & 28 \\
NGC3344 & -19.13 & 0.52 & -22.00 & 0.34 & 0 \\
NGC4395 & -17.68 & 0.43 & -18.27 & 0.18 & 23 \\
UGC8833 & -11.07 & 0.42 & -10.00 & 0.14 & 78 \\
LeoI & -11.05 & 0.41 & -10.00 & 0.08 & 111 \\
M95 & -19.44 & 0.41 & -23.18 & 0.10 & 10 \\
M96 & -19.97 & 0.40 & -23.65 & 0.06 & 96 \\
SextansB & -13.91 & 0.41 & -10.00 & 0.07 & 22 \\
NGC4826 & -20.19 & 0.45 & -24.04 & 0.20 & -132 \\
M66 & -20.35 & 0.41 & -23.98 & 0.09 & -28 \\
DDO187 & -12.71 & 0.44 & -10.00 & 0.17 & 3 \\
GR8 & -12.16 & 0.47 & -10.00 & 0.25 & -18 \\
Sextans & -8.10 & 0.40 & -10.00 & 0.05 & -6 \\
SextansA & -13.93 & 0.41 & -10.00 & 0.07 & 1 \\
NGC3115 & -20.26 & 0.41 & -24.05 & 0.09 & -163 \\
KKH86 & -10.40 & 0.42 & -10.00 & 0.13 & 18 \\
NGC2784 & -19.59 & 0.55 & -23.64 & 0.38 & -251 \\
NGC3109 & -15.50 & 0.40 & -16.32 & 0.09 & 9 \\
NGC5068 & -19.11 & 0.55 & -21.65 & 0.38 & 0 \\
Antlia & -9.75 & 0.43 & -10.00 & 0.16 & -46 \\
NGC3621 & -19.21 & 0.41 & -22.44 & 0.11 & -5 \\
PGC48111 & -13.65 & 0.44 & -10.00 & 0.19 & 53
\end{tabular}


Table 4-Continued

\begin{tabular}{lrrrrl}
\hline \hline \multicolumn{1}{c}{ Name } & $M_{B}$ & $\Delta M_{B}$ & $M_{K}$ & $\Delta M_{K}$ & Peculiar RV \\
\hline KK98-112 & -12.31 & 0.44 & -10.00 & 0.18 & -2 \\
IC4316 & -13.49 & 0.46 & -10.00 & 0.23 & 153 \\
KK98-208 & -14.24 & 0.44 & -10.00 & 0.19 & -163 \\
M83 & -20.24 & 0.43 & -23.53 & 0.16 & -1 \\
KK98-200 & -11.96 & 0.43 & -10.00 & 0.15 & -67 \\
NGC5264 & -15.90 & 0.48 & -17.74 & 0.27 & -53 \\
NGC5253 & -16.93 & 0.42 & -19.31 & 0.15 & -49 \\
PGC39032 & -12.75 & 0.43 & -10.00 & 0.17 & 104 \\
NGC5102 & -17.54 & 0.47 & -20.74 & 0.25 & -19 \\
PGC47171 & -15.42 & 0.47 & -10.00 & 0.25 & -1 \\
NGC5128 & -20.53 & 0.43 & -23.93 & 0.16 & 28 \\
PGC48738 & -14.04 & 0.47 & -10.00 & 0.25 & 58 \\
NGC5408 & -16.51 & 0.44 & -17.02 & 0.22 & -55 \\
NGC4945 & -19.53 & 0.75 & -23.58 & 0.64 & 0 \\
PGC51659 & -11.83 & 0.45 & -10.00 & 0.20 & -81 \\
ESO274G01 & -17.77 & 0.68 & -20.00 & 0.56 & 0 \\
Circinus & -19.55 & 1.04 & -22.18 & 0.97 & 0 \\
MilkyWay & -20.50 & 0.48 & -23.70 & 0.57 & -49 \\
IC3104 & -14.85 & 0.44 & -13.18 & 0.39 & -3 \\
NGC2915 & -15.82 & 0.48 & -18.06 & 0.26 & -78 \\
PGC20125 & -14.76 & 0.46 & -10.00 & 0.22 & -67 \\
Carina & -9.24 & 0.47 & -10.00 & 0.24 & -64 \\
LMC & -17.92 & 0.40 & -10.00 & 0.00 & 8 \\
IC5052 & -18.16 & 0.56 & -20.22 & 0.40 & 0 \\
SagDIG & -10.13 & 0.41 & -10.00 & 0.10 & -60 \\
SMC & -16.35 & 0.40 & -10.00 & 0.00 & -44 \\
PGC19337 & -14.78 & 0.46 & -10.00 & 0.23 & -14 \\
Tucana & -9.13 & 0.43 & -10.00 & 0.15 & -14 \\
NGC1313 & -19.35 & 0.40 & -20.51 & 0.08 & -15 \\
NGC6822 & -15.12 & 0.41 & -16.69 & 0.10 & 20 \\
NGC1705 & -15.81 & 0.48 & -18.02 & 0.27 & 58 \\
& & & & &
\end{tabular}


Table 4-Continued

\begin{tabular}{lrrrrl}
\hline \hline \multicolumn{1}{c}{ Name } & $M_{B}$ & $\Delta M_{B}$ & $M_{K}$ & $\Delta M_{K}$ & Peculiar RV \\
\hline IC5152 & -15.42 & 0.46 & -17.10 & 0.23 & -63 \\
PGC09962 & -15.21 & 0.46 & -14.77 & 0.29 & 20 \\
KK98-54 & -13.17 & 0.48 & -10.00 & 0.26 & -61 \\
DDO210 & -11.09 & 0.41 & -10.00 & 0.07 & -59 \\
Phoenix & -10.01 & 0.40 & -10.00 & 0.05 & -136 \\
PGC01641 & -10.84 & 0.41 & -10.00 & 0.10 & -50 \\
ESO245-05 & -15.60 & 0.46 & -10.00 & 0.22 & 16 \\
NGC55 & -17.83 & 0.65 & -20.36 & 0.52 & -31 \\
NGC625 & -16.31 & 0.42 & -18.86 & 0.13 & 68 \\
UGCA438 & -12.88 & 0.41 & -10.00 & 0.10 & -46 \\
NGC300 & -17.63 & 0.41 & -20.15 & 0.10 & -21 \\
UGCA442 & -14.62 & 0.48 & -15.38 & 0.43 & 17 \\
NGC7793 & -18.06 & 0.46 & -21.10 & 0.24 & -5 \\
Sculptor & -9.49 & 0.42 & -10.00 & 0.11 & 90 \\
Fornax & -11.47 & 0.43 & -10.00 & 0.14 & -39 \\
NGC253 & -20.02 & 0.45 & -24.21 & 0.20 & 20 \\
IC1574 & -13.43 & 0.47 & -10.00 & 0.26 & 94 \\
DDO6 & -12.50 & 0.43 & -10.00 & 0.16 & 140 \\
NGC247 & -17.75 & 0.52 & -20.10 & 0.34 & 10 \\
WLM & -13.88 & 0.41 & -10.00 & 0.10 & -62 \\
IC1613 & -14.54 & 0.40 & -11.55 & 0.12 & -122 \\
DDO216 & -11.48 & 0.49 & -10.00 & 0.29 & 26 \\
UGC685 & -14.42 & 0.42 & -10.00 & 0.14 & 59 \\
NGC628 & -19.67 & 0.60 & -22.48 & 0.45 & 370 \\
AndVI & -10.54 & 0.41 & -10.00 & 0.08 & -124 \\
LGS3 & -9.94 & 0.41 & -10.00 & 0.07 & -82 \\
KK98-16 & -12.38 & 0.47 & -10.00 & 0.24 & 114 \\
M33 & -18.44 & 0.40 & -20.43 & 0.07 & 10 \\
KK98-17 & -11.41 & 0.44 & -10.00 & 0.19 & 64 \\
AndII & -10.87 & 0.40 & -10.00 & 0.03 & 28 \\
AndIII & -9.65 & 0.45 & -10.00 & 0.20 & -113 \\
& & & & &
\end{tabular}


Table 4-Continued

\begin{tabular}{lccccl}
\hline \hline Name & $M_{B}$ & $\Delta M_{B}$ & $M_{K}$ & $\Delta M_{K}$ & Peculiar RV \\
\hline NGC404 & -16.57 & 0.42 & -18.96 & 0.15 & 11 \\
KKH98 & -10.78 & 0.41 & -10.00 & 0.11 & 15 \\
AndI & -11.04 & 0.41 & -10.00 & 0.07 & -132 \\
NGC925 & -19.44 & 0.40 & -21.06 & 0.05 & 169 \\
M31 & -20.28 & 0.40 & -23.40 & 0.06 & -56 \\
NGC205 & -15.65 & 0.45 & -18.71 & 0.21 & 6 \\
KKH18 & -12.39 & 0.47 & -10.00 & 0.24 & 112 \\
NGC891 & -19.08 & 0.42 & -23.67 & 0.14 & 219 \\
NGC185 & -14.65 & 0.45 & -17.40 & 0.22 & 60 \\
NGC147 & -14.68 & 0.49 & -17.20 & 0.29 & 62 \\
KKH96 & -11.73 & 0.41 & -10.00 & 0.11 & -33 \\
KKH5 & -12.27 & 0.43 & -10.00 & 0.17 & 74 \\
IC10 & -18.89 & 0.48 & -18.09 & 0.26 & -80 \\
MaffeiI & -20.04 & 0.45 & -22.71 & 0.22 & 129 \\
Maffei2 & -20.16 & 1.01 & -22.02 & 0.93 & 57 \\
\hline
\end{tabular}

Note. - Derived absolute magnitudes of Local Volume galaxies in $B$ and $K$, with uncertainties taking into account distance and photometric errors; along with the peculiar radial velocities relative to the best-fit isotropic expansion. Galaxies for which no photometric data were available were arbitrarily assigned an absolute magnitude of -10 . Their listed uncertainties, which are not used in any calculations, show the effect of distance uncertainties alone. 
As will become clearer below, it is important to know how far to trust the derived peculiar velocities. They are uncertain because of the uncertainties in the model parameters, and because of uncertainties in distance (uncertainties in angle having negligible effect). For situations in which a derived quantity $Q$ is a function of various independent parameters $p_{i}$, each with an uncertainty $\delta p_{i}$, I have used the formula

$$
(\delta Q)^{2}=\sum_{i}\left(\frac{\partial Q}{\partial p_{i}} \delta p_{i}\right)^{2}
$$

which is sufficiently accurate as long as the errors are not large fractions of the values. For the uncertainties in peculiar velocities due to model parameter uncertainties and distance errors $(\delta D)$ this technique gives

$$
\left(\delta p v_{i}\right)^{2}=x_{i}^{2} \delta V_{x}^{2}+y_{i}^{2} \delta V_{y}^{2}+z_{i}^{2} \delta V_{z}^{2}+D_{i}^{2} \delta H^{2}+H^{2} \delta D^{2}
$$

And for the uncertainties in absolute magnitude $M$ due to errors in apparent magnitude $m$ and distance $D$,

$$
\delta M^{2}=\delta m^{2}+\left(\frac{5}{\ln 10} \frac{\delta D}{D}\right)^{2}
$$

All $B$ photometry is assigned a $\delta m$ of 0.4 magnitude, while $K$ uncertainties are taken from the source. The results are listed in Table (4). The uncertainties in absolute magnitude for those galaxies without photometry reflects errors in distances alone, and are left in the table to give an idea of the relative importance of distance and photometry uncertainties.

\subsection{Peculiar Velocity Dispersions: Bright Galaxies}

We now turn to smaller-scale motions, those which can be connected with individual galaxies or galaxy groups. For this the dynamical youth of the Local Volume is invoked: given the peculiar velocity dispertion of $70-80 \mathrm{~km} \mathrm{~s}^{-1}$, we expect that satellite galaxies around a mass concentration to be mostly still infalling.

To look for this, we pick out the brightest galaxies, those with $M_{B}$ of -20 or brighter. (While one can argue about the role of starbursts, for example, in skewing the mass-to-light ratio, it is certain that $M_{B}$ of -20 does select the very biggest concentrations of luminous matter in the Local Volume. They are also the brightest in $K$.) We turn our attention to those galaxies between 0.5 and $1.5 \mathrm{Mpc}$ away from the bright ones, to isolate a sample which should feel their gravity but which probably haven't crossed from one side to the other (near to far, or vice versa). Finally, we choose only those satellites in front of or behind the giants 
(with the relative direction within 60 degrees of the line of sight), so that any motion due to infall shows up well in radial velocity.

To see the order of magnitude of the effect we are looking for, consider a galaxy of $10^{12}$ solar masses, acting at a distance of $1 \mathrm{Mpc}$ over 10-13 billion years. That results in a change in speed of 44-67 $\mathrm{km} \mathrm{s}^{-1}$; so we look for something in the tens, but not hundreds, of $\mathrm{km}$ $\mathrm{s}^{-1}$. The average of satellites behind the giant galaxy should show a blueshift (negative), and those before a redshift; the difference should be on the order of $100 \mathrm{~km} \mathrm{~s}^{-1}$. There should be some noise from those satellites having made a close approach, but given the observed peculiar velocities we do not expect very many of them. There are not enough satellite galaxies around most of the bright ones to give a clear picture, so the data from all groups outside the Local Group are averaged together in Table (5). There being no satellites "before" the Milky Way, and only one for M31, the numbers listed for those giants are all "behind". 
Table 5. Satellite Galaxy Motions

\begin{tabular}{lcccc}
\hline \hline \multicolumn{1}{c}{ Quantity } & Before & Behind & M31 & Milky Way \\
\hline & $\mathrm{km} \mathrm{s}^{-1}$ & $\mathrm{~km} \mathrm{~s}^{-1}$ & $\mathrm{~km} \mathrm{~s}^{-1}$ & $\mathrm{~km} \mathrm{~s}^{-1}$ \\
Mean & $10 \pm 27$ & $-39 \pm 3$ & $-74 \pm 85$ & $-73 \pm 37$ \\
rms dispersion & 61 & 107 & 76 & 66 \\
Number of satellites & 7 & 17 & 10 & 24 \\
\hline
\end{tabular}

Note. - Satellite galaxy peculiar radial velocities relative to bright $\left(M_{B}<-20\right)$ galaxies, from the combined Local Volume sample. Satellites are between 0.5 and $1.5 \mathrm{Mpc}$ from the giant, and sorted into those in front and those behind; those at a similar distance are not included. The satellites of M31 and the Milky Way are numerous enough to show separately; all these are "behind", in the sense that infall would show as a negative difference in peculiar velocity; except for Carina in the case of M31, which is not included here. 
In half of the cases there is no significant sign of infall. In another (the Volume average "behind") the rms dispersion is much larger than the average, so infall contributes only a minor part of the peculiar velocity. There is a fairly clear sign of infall into the Milky Way. But if an effect is seen in only half (or less) of the places it should be it can hardly be accepted as a general feature of the situation, and there is the strong possibility that the positive signs are due to something else.

The method of computing the uncertainties here is that used for averages of data points, each of which has an assigned uncertainty $\sigma_{i}$; which uncertainties are uncorrelated with each other. I have used the following formula for the uncertainty of the average, $\sigma_{T}$ :

$$
\frac{1}{\sigma_{T}^{2}}=\sum_{i} \frac{1}{\sigma_{i}^{2}} .
$$

Now, the uncertainties among satellite galaxy peculiar velocities are correlated, since the largest part comes from parameters in the model and changing a parameter for one will change the parameter for all. That would require, formally, that uncertainties be added, resulting in a much larger overall uncertainty. On the other hand, for satellites about the same giant any change in parameters will affect them all in much the same way, causing less overall uncertainty. Rather than spend more time on this rather marginal calculation attempting to sort out uncertainties, however, I will note that the rms dispersions alone show that infall is not a strong signal, even if present; and treat the effects of changes in the model in more detail in a later section.

These results are surprising not least in the fact that Karachentsev et al. (2003a) and Karachentsev et al. (2003b) found frontside infall but no backside infall in Local Volume groups. Most of this I will trace to differences in the background model, of which (again) I postpone a detailed discussion for a later section. Part could be due to spurious signals in poor or limited data. As examples of the latter there is the peculiar velocity versus Supergalactic Z plot, Figure 6 in Karchentsev \& Makarov (1996), which shows a clear signal of infall into the Supergalactic Plane, a signal which disappears with more and better data; and the lopsided histogram of peculiar velocities of Whiting (2003), which led to the conclusion that many nearby galaxies of high radial velocity were being missed-a conclusion which also disappears with the present collection of data.

Additionally, consider Figure (1). It is tempting to see in it an increase in peculiar velocity dispersion around $4 \mathrm{Mpc}$, due to the M81 and Centaurus A groups.

In fact there is no significant increase in peculiar velocity dispersion. Within $1.5 \mathrm{Mpc}$ the dispersion is $70 \mathrm{~km} \mathrm{~s}^{-1}$; from 3 to $5 \mathrm{Mpc}, 84 \mathrm{~km} \mathrm{~s}^{-1}$, which is statistically indistinguishable (with this number of data points). (Note that both are indistinguishable from the peculiar 
velocity dispersion of the Volume as a whole.) There are more points on the plot at the larger distance, hence the distribution is more fully shown including the wings; but it is essentially the same distribution. Similarly, there is a diagonal line of points between about 1.5 and $2.5 \mathrm{Mpc}$, which could easily be taken as a sign of front side infall into Centaurus or M81. But this is made up of galaxies in at least four widely-separated parts of the sky, so attributing a coordinated motion to M81 or Centaurus is questionable; and anyway the structure disappears when error bars are introduced (as in the right panel of Figure (1)).

So it is at least plausible that apparently clear signs of infall seen in other investigations are mistaken. We now turn to other ways of relating peculiar motions to luminous matter.

Perhaps the assumption of dynamical youth implicit in the idea of infall is not right, at least close to the giants. If these regions are dynamically old, that is if the groups are virialized, then there should be a much larger velocity dispersion close to the giant compared with farther out. (It should also be true more generally that satellite galaxies deeper inside a potential well show higher speeds, as potential energy is converted to kinetic.) To look for this we choose two zones, an inner zone out to $1 \mathrm{Mpc}$ from the giant and an outer zone from 1 to $2 \mathrm{Mpc}$ away. The resulating velocity dispersions are shown in Table (6). Included is an F-ratio calculation, which shows the probability of the outer sample being drawn from a significantly different (smaller) parent distribution. 

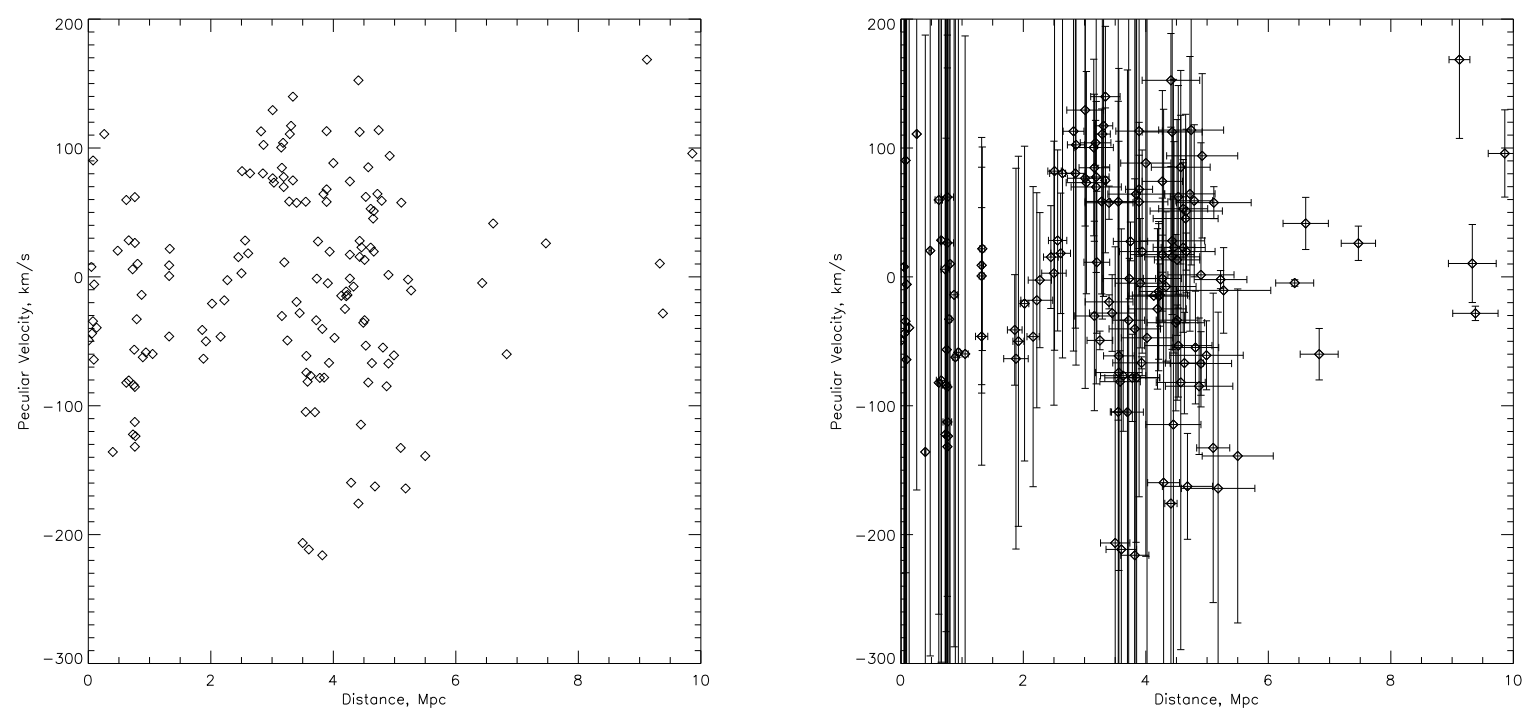

Fig. 1.- Peculiar velocity as a function of distance (for the isotropic model). Left, without error bars to show apparent structure; right, with error bars.

Table 6. Satellite Galaxy Velocity Dispersions

\begin{tabular}{lccc}
\hline \hline \multicolumn{1}{c}{ Subsample } & Inner & Outer & Significance \\
\hline & $\mathrm{km} \mathrm{s}^{-1}$ & $\mathrm{~km} \mathrm{~s}^{-1}$ & \\
non-Local Group & 114 & 108 & 0.64 \\
M31 & 83 & 64 & 0.83 \\
Milky Way & 94 & 69 & 0.82 \\
\hline
\end{tabular}

Note. - Velocity dispersion of satellites around bright galaxies in the Local Volume (rms difference from the giant), for various subsamples. Inner galaxies are less than $1 \mathrm{Mpc}$ from the giant, outer satellites between 1 and $2 \mathrm{Mpc}$. The last column shows the significance level at which the hypothesis of equal inner and outer dispersions is rejected by the F-ratio test. 
There does seem to be a smaller velocity dispersion farther from the giants, but it's not even of one-sigma significance outside the Local Group, and not much better within. (These should probably be taken as upper limits on the significance, since the satellite galaxy dispersions are not really Gaussian.) How much should we see?

Prada et al. (2003) examined isolated giant and satellite galaxy pairs in the SDSS data, calculating the line of sight velocity dispersion for 3000 satellites at various distances from the primary. For an $\mathrm{L}^{*}$ galaxy (which is comparable to those examined above), the dispersion fell from $120 \mathrm{~km} \mathrm{~s}^{-1}$ at $20 \mathrm{kpc}$ to $60 \mathrm{~km} \mathrm{~s}^{-1}$ at $350 \mathrm{kpc}$. At this rate the giantsatellite dispersion would be a minor part of the dispersion as observed in the Local Volume at megaparsec scales. In all, it is unlikely that we have observed any influence of large galaxies on the velocity dispersion of satellites between 1 and $2 \mathrm{Mpc}$ and much more likely that what has been observed is due to some other effect. (If we separate the sample from beyond the Local Group into "behind and before" and "beside", the inner and outer "beside" samples have the same dispersion to within one $\mathrm{km} \mathrm{s}^{-1}$; which, at $120 \mathrm{~km} \mathrm{~s}^{-1}$, is larger than the "before and behind" inner sample. But selecting data in this way can have a pernicious effect on statistics, and at the very least increases the chance that small-number fluctuations will give misleading results.)

\subsection{The Peculiar Gravity Field}

So far we have dealt with only a subset of our data, those galaxies near the very brightest. If we wish to make truly general comparisons of peculiar velocities with luminous matter, as well as make use of a great deal of data painstakingly gathered, we should find a way to look at all the galaxies in the sample.

For this we again make use of the dynamical youth of the Local Volume, which allows us to compare apples with oranges. Assume that peculiar velocities are produced by fluctuations in the gravitational field which can be identified with galaxies. If the fluctuations are linear (as they are on much larger scales than we are looking at), the local gravitational acceleration and the local peculiar velocity should be proportional: plotting one against the other gives a straight line. In the mildly nonlinear regime the line will become an S-shaped curve, as the effects of mass concentrations reinforce themselves. As some objects complete infall and pass to the other side of larger masses, the ends of the $\mathrm{S}$ will fray; but as long as the system is not dynamically relaxed there will be some discernable relation between the gravity field and the peculiar velocity field: apples and oranges.

If possible, we want to avoid the worst inaccuracies in this kind of calculation. Galaxies 
which are at similar distances and close to each other in the sky will have large calculated gravitational accelerations, but distance errors as well as motions mean that they may actually be in front when the calculation has them behind, and vice versa. To reduce this problem we adopt a smoothing parameter $A$, which is set to the distance an object with a speed of $78 \mathrm{~km} \mathrm{~s}^{-1}$ will go in the age of the universe, about $1.2 \mathrm{Mpc}$ (setting this parameter to anything between 1 and 2 Mpc makes little difference).

We proceed, then, by finding the smoothed gravitational acceleration at each of our galaxies, using the luminosity weights $w_{B}$ or $w_{K}$, and the smoothing length $A$; then find the component in the radial direction $\hat{\mathbf{r}}$ :

$$
g_{i}=\mathbf{g}_{i} \cdot \hat{\mathbf{r}}_{i}=G \sum_{j \neq i} w_{j} \frac{\mathbf{r}_{j}-\mathbf{r}_{i}}{\left(\left(\mathbf{r}_{j}-\mathbf{r}_{i}\right)^{2}+A^{2}\right)^{3 / 2}} \cdot \hat{\mathbf{r}}_{i}
$$

This we shall call the synthetic gravity, or sometimes the synthetic peculiar gravity. There is a normalization constant implied in the weights $w_{j}$, which depends on details of dynamical model one prefers. For the moment we leave the synthetic gravity in arbitrary numbers.

When calculated this way, the radial components of the gravitational force become steadily more negative as we proceed to the outskirts of the Volume. This is as expected. The average mass density of the Volume will exert a force inward, increasing linearly with distance from the center. Since we are interested in deviations from the average, however, we fit a linear force to the calculation above and subtract it.

Of course, the uncertainty in the synthetic gravity is important. These will be due to errors in mass weight $w_{j}$ (proportional to $\delta M$ above) and distances $r_{j}$ and $r_{i}$, and using the formulation in Equation(3) we have

$$
\begin{aligned}
\delta g_{i}^{2} & =\sum_{j \neq i}\left[\left(\frac{r_{j} \hat{\mathbf{r}}_{j} \cdot \hat{\mathbf{r}}_{i}-r_{i}}{\left(\left(\mathbf{r}_{j}-\mathbf{r}_{i}\right)^{2}+A^{2}\right)^{3 / 2}} \delta w_{j}\right)^{2}\right. \\
& \left.+\left(\frac{w_{j} \hat{\mathbf{r}}_{j} \cdot \hat{\mathbf{r}}_{i}}{\left(\left(\mathbf{r}_{j}-\mathbf{r}_{i}\right)^{2}+A^{2}\right)^{3 / 2}}-3 \frac{w_{j}\left(r_{j} \hat{\mathbf{r}}_{j} \cdot \hat{\mathbf{r}}_{i}-r_{i}\right)\left(r_{j}-r_{i} \hat{\mathbf{r}}_{j} \cdot \hat{\mathbf{r}}_{i}\right)}{\left(\left(\mathbf{r}_{j}-\mathbf{r}_{i}\right)^{2}+A^{2}\right)^{5 / 2}}\right)^{2} \delta r_{j}^{2}\right] \\
& +\left[\sum_{j \neq i}\left(\frac{-w_{j}}{\left(\left(\mathbf{r}_{j}-\mathbf{r}_{i}\right)^{2}+A^{2}\right)^{3 / 2}}+3 w_{j} \frac{\left(r_{j} \hat{\mathbf{r}}_{j} \cdot \hat{\mathbf{r}}_{i}-r_{i}\right)^{2}}{\left(\left(\mathbf{r}_{j}-\mathbf{r}_{i}\right)^{2}+A^{2}\right)^{5 / 2}}\right)\right]^{2} \delta r_{i}^{2}
\end{aligned}
$$

where $\hat{\mathbf{r}}_{j}$ is the unit vector in the $\mathbf{r}_{j}$ direction, and similarly.

The resulting plot for $B$ luminosity is shown in the left panel of Figure (2). There is no apparent linear, quasi-linear or frayed-S shape. In fact there is no apparent correlation at 
all. Postponing for a moment seeking a correlation with more subtle statistical tools than the human eye, we will first try to find a plot which gives something more obvious.

Perhaps the problem lies in using blue luminosities. It is well known that starbursts can skew the mass-to-light ratio in this band drastically; the nearby dwarf IC 10 is nearly as luminous as the spiral M33 in $B$, but much fainter in the infrared. Using $K$-band luminosity, which should be a much better measure of the actual mass of the galaxies, we arrive at the right panel of Figure (2).

There is, again, no visible correlation. There are significantly fewer galaxies with $K$ photometry to contribute to the synthetic gravity field, but the effect of the unmeasured objects should be slight: none are as bright as the Magellanic Clouds in $B$, and so the combined effect of all of them is less than about that of one giant galaxy.

Recalling that the anisotropic model was calculated to be a better fit to the data, we turn to the peculiar velocities based on that model for Figure (3). Again there is no improvement apparent.

Perhaps the uncertainties in the various quantities are large enough to obscure any correlation; that is, perhaps the data are simply not good enough yet to see a signal. Adding the uncertainties in synthetic gravity and peculiar velocity as error bars gives Figure (4).

Some points on the plots are clearly very uncertain indeed. Though it appears that there are enough good data to delineate a trend, if it existed, including all the errors serves to obscure the matter more than it helps. Using only the 63 points with the best synthetic gravity uncertainties, we have Figure (5). As one would expect, points have been preferentially removed at synthetic gravities of large (positive and negative) magnitude, since these are most subject to distance errors in nearby galaxies; and there is a bit of structure due to small-number statistics. However, no real trend is visible, and the errors here are certainly small enough to show one.

I have claimed that there is no correlation on any of the plots presented; however, it is very difficult to prove the absence of any correlation. (In fact there could be a very strong correlation of a particular kind, say a high-frequency sine wave, which would be entirely masked by the errors. But we are looking for something less general, a monotonic function.) The fact that none is evident in the plots here presented is a strong indication in that direction, the human eye being very good at detecting correlations amidst noise (indeed, even when none exists); but we would like something quantitative.

Consider a linear relation. While we expect the Local Volume to be nonlinear as far as gravitational effects are concerned, we should certainly be able to approximate it with a 

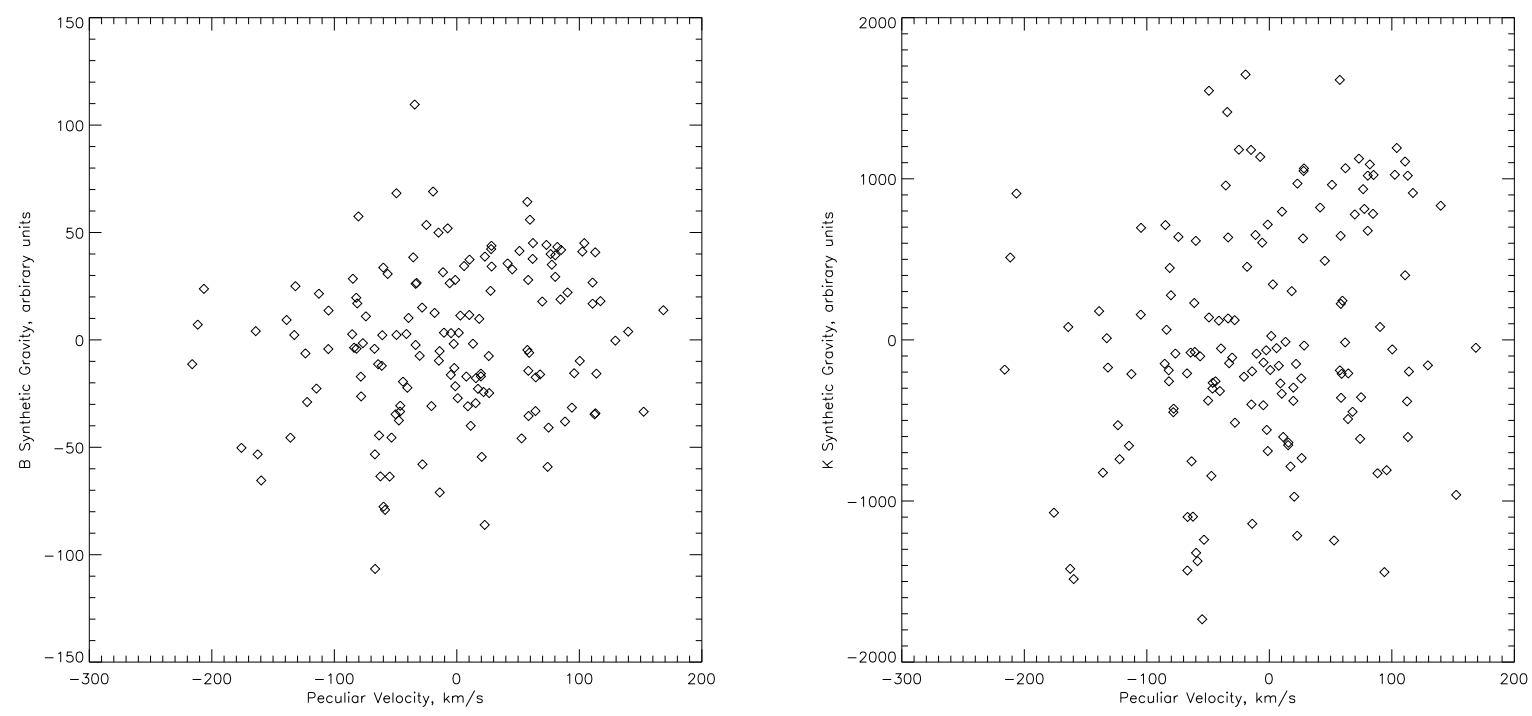

Fig. 2.- Peculiar radial velocities of galaxies within the Local Volume (as calculated from the isotropic-expansion kinematic model) plotted against the radial component of gravitational force, assuming a constant ratio of mass to light in the $B$ band (left) and $K$ band (right).
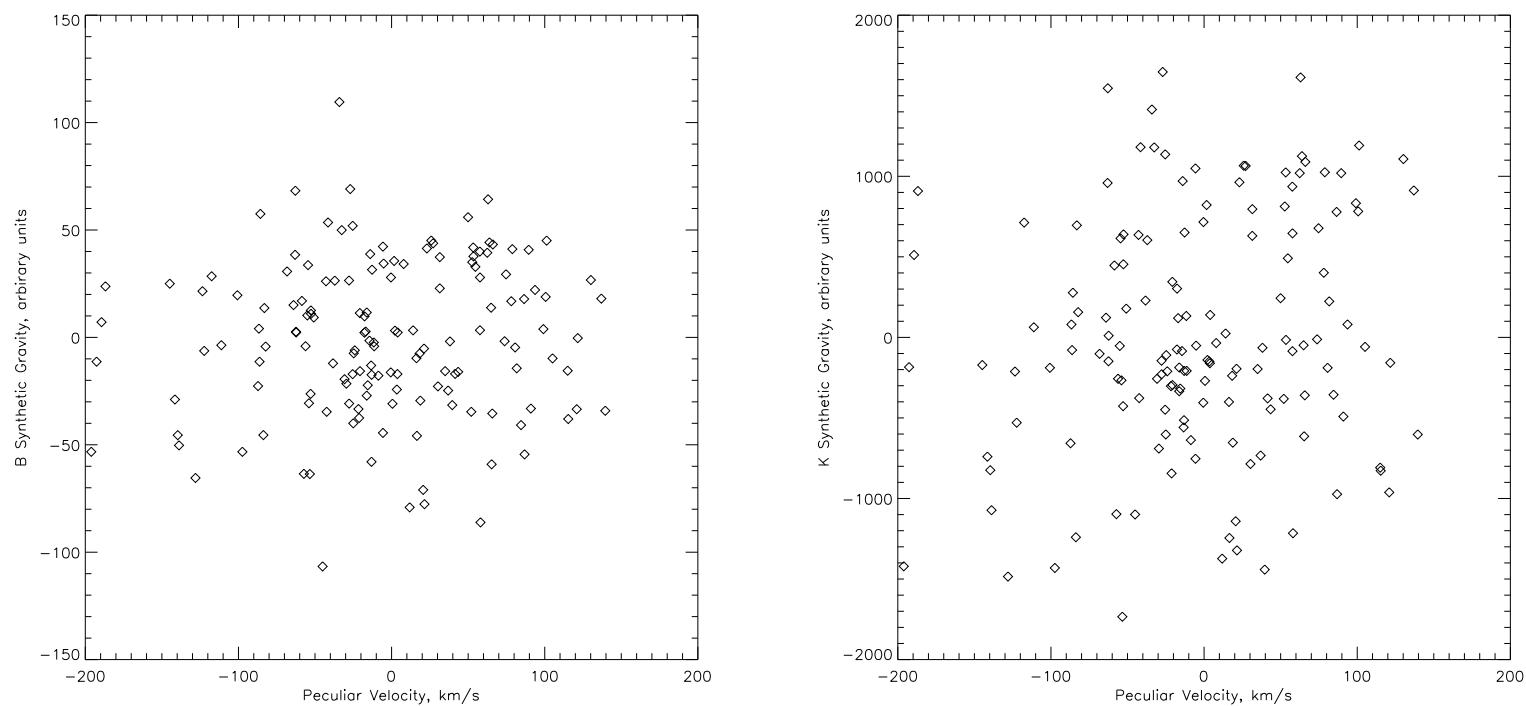

Fig. 3.- Peculiar radial velocities of galaxies within the Local Volume plotted against the reconstructed radial gravitational acceleration as before, but using the tensor (anisotropic expansion) solution. Again, $B$ band light is used on the left, $K$ band on the right. 

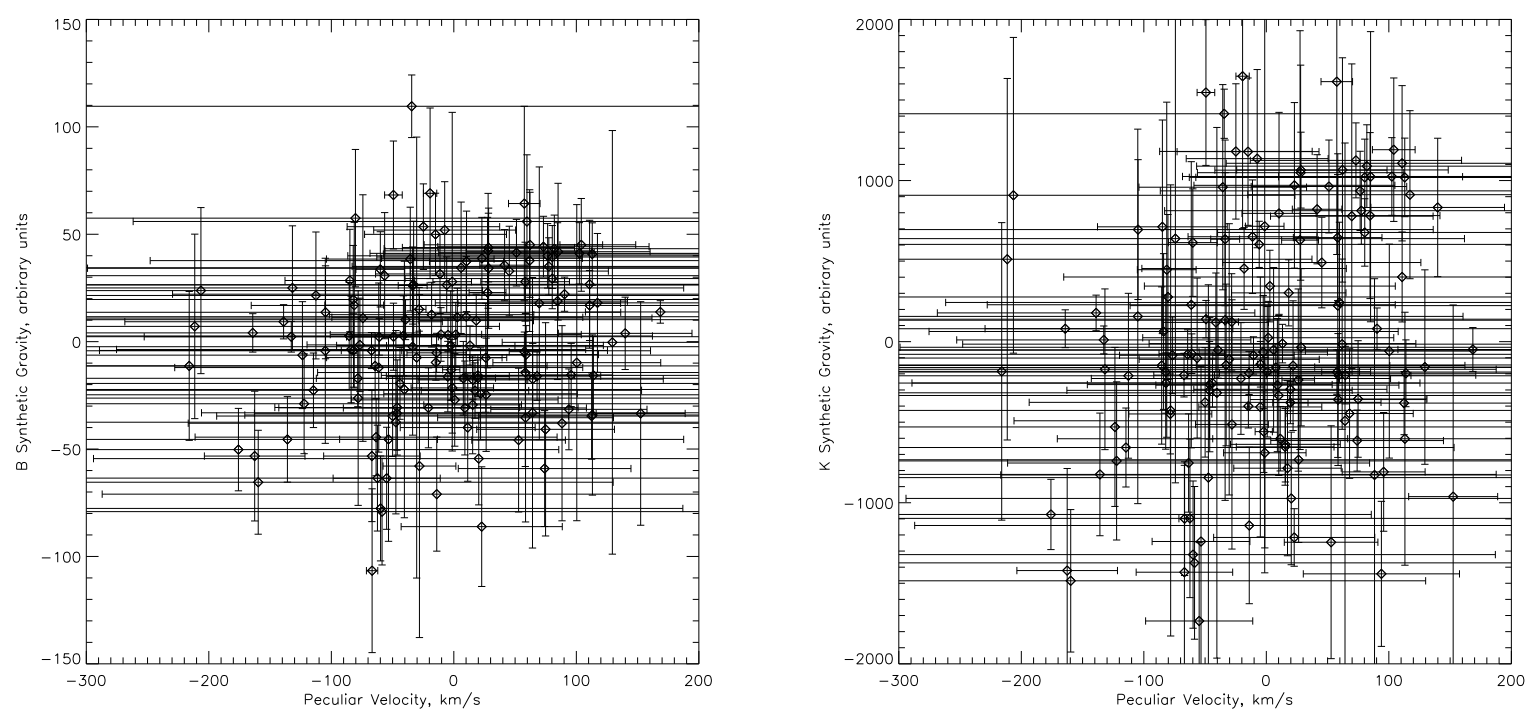

Fig. 4.- As in Figure 2, but with error bars included. Uncertainties in peculiar velocity are calculated from those in the model parameters and galaxy distances; those in synthetic gravity, from uncertainties in distances and photometry.
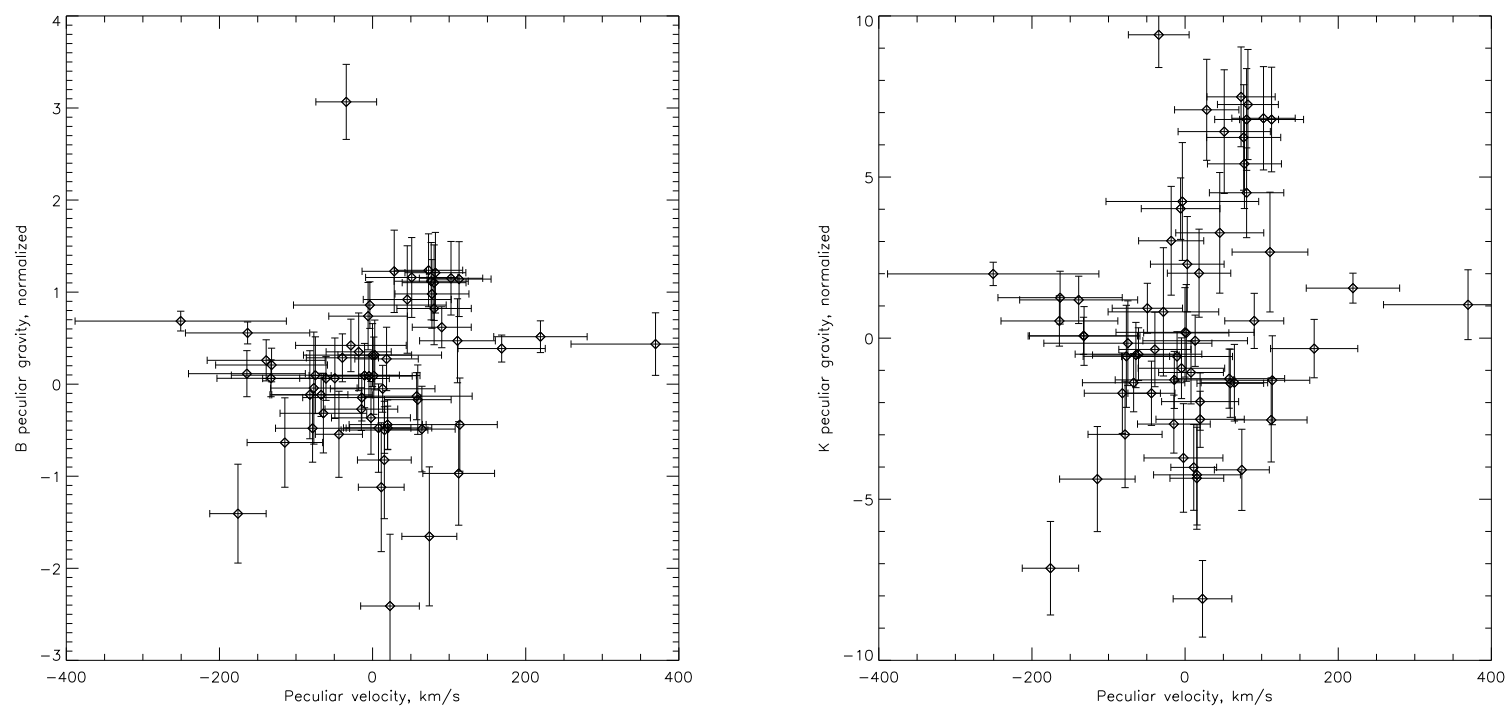

Fig. 5.- As in Figure 4, but including only the 63 points with the smallest error bars. 
straight line to some degree of accuracy. If we fit one to the $K$-band data ${ }^{2}$ of Figure (2), then subtract the fit, we are left with a peculiar velocity dispersion of $77 \mathrm{~km} \mathrm{~s}^{-1}$. This is indistinguishable, statistically, from the raw dispersion; so there is no linear fit.

In search of the most basic, qualitative signal, we then divide the plot into positive and negative peculiar gravity halves, excluding any points whose errors would take them across the zero line. For each half we form the average and estimate the uncertainty in the average based on the individual uncertainties in the peculiar velocities. For the region of positive $K$ peculiar gravity, the average peculiar radial velocity is $+27 \mathrm{~km} \mathrm{~s}^{-1} \pm 7$; for negative, -23 $\mathrm{km} \mathrm{s}^{-1} \pm 6$. The rms velocity dispersion for both positive and negative $\mathrm{K}$ is $49 \mathrm{~km} \mathrm{~s}^{-1}$. But again, the uncertainties in peculiar velocity are correlated, so the stated errors could be very misleading; and the dispersions are much larger than the averages in magnitude. There could be a general, average correlation of peculiar velocity with synthetic gravity, but at best it explains a minority of the actual motion, and it is not certain it exists.

It is time to examine the model itself in terms of its dynamical implications.

\subsection{A Dynamical Background Model}

Up to this time we have used the average, kinematically-derived background model as a basis for peculiar velocities, and have found no correlation between velocities (apples) and luminosity-derived peculiar gravity (oranges). Suppose we try a comparison which adjusts the model to minimize the difference between the peculiar velocity and the $K$-band synthetic gravity:

$$
\sigma^{2}=\frac{1}{N} \sum_{i}\left(g_{i}-v_{p i}\right)^{2}=\frac{1}{N} \sum_{i}\left(g_{i}-\left(v_{\mathrm{obs}}-\left(\mathbf{v}_{0} \cdot \hat{\mathbf{r}}+\hat{\mathbf{r}} \cdot \mathbf{H} \cdot \mathbf{r}\right)\right)\right)^{2}
$$

We are faced with the fact that the normalization of the synthetic gravity, $g_{i}$, is unknown. If it is too small, we are essentially reproducing the kinematic model; if it is too big, we are fitting a model to the luminosity field and ignoring motions - a calculation which might be of some interest, but not to us now.

In practice a series of models was calculated with different normalizations, starting with one which matched the rms value of the peculiar velocity in the isotropic kinematic model. This turned out in fact to give the best correlation, shown in the left-hand panel of Figure

\footnotetext{
${ }^{2}$ From here on calculations will be confined to the $K$ band peculiar gravities to save time and space. This band should show the effect of mass best, if any exists, and the incompleteness of our data should not make any significant overall difference.
} 
(6), with parameters shown in Table (7).

Finally we have an undoubted correlation. There is significant scatter, but it is no longer possible to say that there is no relation between luminosity and peculiar velocity. (An anisotropic solution, calculated at the same time, gave an essentially identical scatter.)

How good is the correlation? If we fit a line, as we did for the $K$-pv plot in the kinematic model, then subtract it, we arrive at a velocity dispersion of $175 \mathrm{~km} \mathrm{~s}^{-1}$. This is significantly better than the $209 \mathrm{~km} \mathrm{~s}^{-1}$ of the uncorrected model; the F-ratio test gives a $98 \%$ chance of the smaller dispersion being drawn from a different distribution.

It is still much larger than the dispersion around the best-fit kinematic model. This is worrying; why should a model showing more insight into the physics give a worse overall correlation? Not necessarily troubling, but adding nothing to the situation, is the fact that neither the new $\mathbf{v}_{0}$ nor the difference (the vector added to the kinematic solution to get the new one) points in the direction of anything interesting outside the Local Volume. It would be interesting, say, if the new vector pointed toward Virgo, or the difference vector in the direction of the CMB dipole. Still, surely one cannot find a correlation which isn't there? 

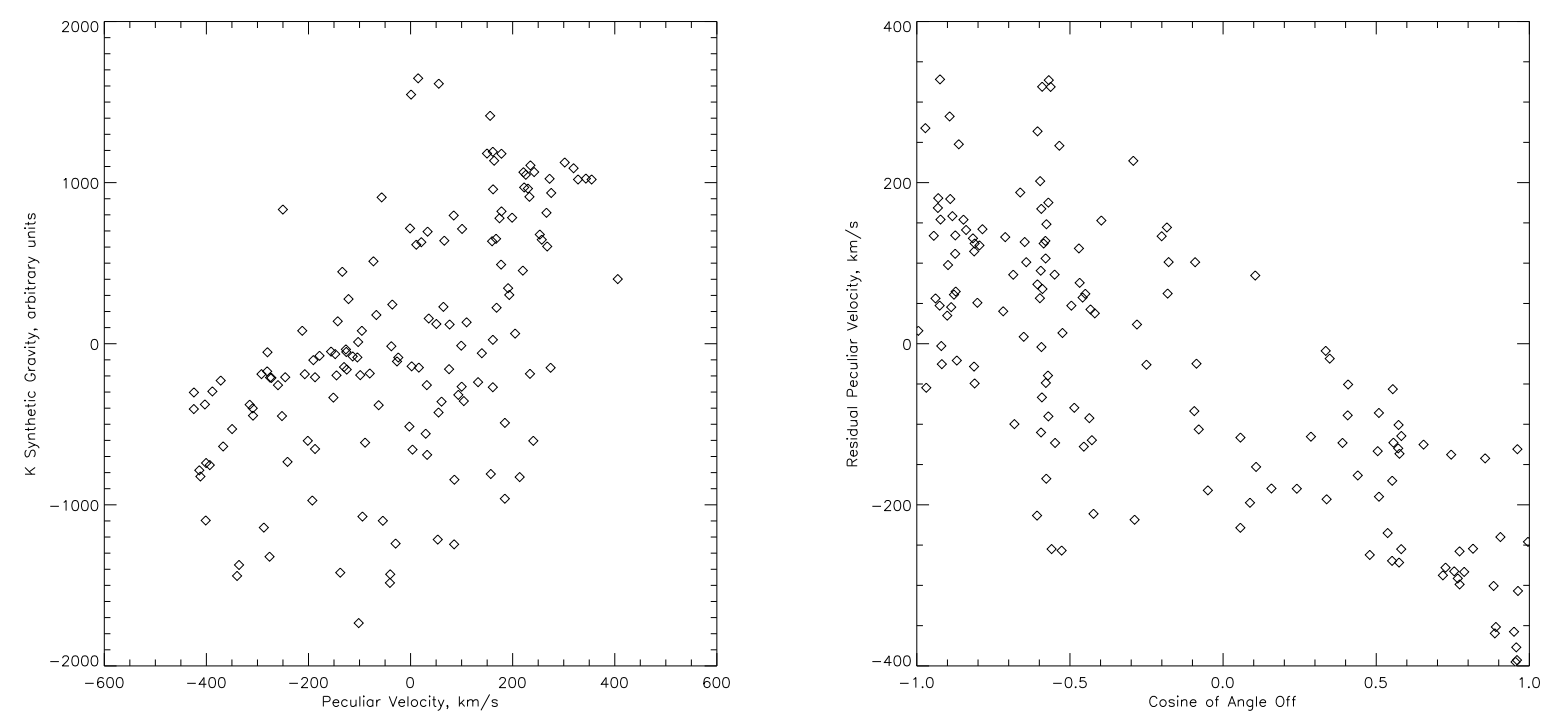

Fig. 6. - Left, the radial component of gravity, assuming mass is proportional to $K$-band light, smoothed on 1.2 Mpc scales, plotted against peculiar velocity relative to a model adjusted to give the best correlation. Right, the residuals of the left-hand plot after a linear fit is subtracted, plotted against the cosine of the angle between the particular galaxy and the difference in constant vectors between the kinematic and dynamic solutions. The clear trend in the right-hand plot shows the trend in the left-hand plot to be spurious; see text.

Table 7. The Dynamical Background Solution

\begin{tabular}{lccc}
\hline \hline \multicolumn{1}{c}{ Quantity } & Magnitude & $\mathrm{L}$ & $\mathrm{B}$ \\
\hline & $\mathrm{km} \mathrm{s}^{-1}$ or $\mathrm{km} \mathrm{s}^{-1} \mathrm{Mpc}^{-1}$ & Degrees & Degrees \\
Dynamical Model & & & \\
$\mathrm{V}$ & 414 & 223 & -20 \\
$\mathrm{H}$ & 91 & & \\
Dynamical minus Kinematic & & 265 & 13 \\
$\mathrm{~V}$ & 325 & & \\
$\mathrm{H}$ & 25 & & \\
\hline
\end{tabular}

Note. - Parameters of the dynamical background model (constant vector plus uniform expansion, maximizing the correlation between peculiar velocity and $K$-band luminosity). 
One can. Consider a Hubble plot of a galaxy group (radial velocity versus distance). Suppose there is no correlation of peculiar velocity with luminosity, so that there is a constant dispersion about the average line. Synthetic gravities will be positive on the near side and negative on the far side, with no evident relation with peculiar velocity.

Next suppose that, instead of the "proper" model, one imposed a higher Hubble constant (steeper slope); now the group as a whole will show negative peculiar velocities. Add a constant vector in the direction away from the group, and all their peculiar velocities will increase. If they increase to the point that the new model fit passes near the center of the group the average peculiar velocity will again be zero, but now there will be more points above the line (positive peculiar velocity) on the near side and more below the line (negative peculiar velocity) on the far side; these will correlate with the synthetic gravity.

If groups were distributed evenly around the sky the better correlation thus gained for one would be offset by a worse correlation for another on the far side. But the distribution of groups in the Local Volume is not even or random, so this could be the explanation for the correlation seen in the left-hand side of Figure (6). There would be three clear signs of this effect: (i) the added constant vector would be in the direction away from most galaxies; (ii) there would be a greater dispersion away from the correlation to negative peculiar velocities (whole groups brought below the Hubble line) than positive; and (iii) there will be a correlation between the residuals (after the spurious correlation is subtracted from peculiar velocities) and the direction of the added constant vector.

Condition (i) is satisfied; the added constant vector is less than $23^{\circ}$ away from the antipode of the average of all radius vectors of Local Volume galaxies in our sample (this has less than $5 \%$ chance of happening at random). Condition (ii) is evident from the lefthand side of Figure(6), and condition (iii) from the right-hand side. Our "correlation" found using the dynamic criterion is spurious. Perhaps more important than this specific result, the sensitive dependence of peculiar velocities on the background model is highlighted.

\section{Discussion and Conclusions}

\subsection{Other Results in the Local Volume}

The main result of this study, that there is no apparent relation between luminosity and peculiar velocity on megaparsec scales in the Local Volume, is at odds with some previous work. Signs of the expected gravitational effects of galaxy groups have been seen and masses have even been calculated. The disagreement is mostly due to the fact that two different questions have been asked. Other work has asked, "What is the mass of this galaxy group?" 
while I have asked, "Is there a detectable mass?" It should not be surprising that different questions come up with different answers.

Additionally, the significance of the fact that one sees an expected sign of the effect of luminous mass on peculiar velocity depends greatly on the context. It is entirely fair and correct to note in passing that a feature on a plot has a ready explanation under plausible assumptions. However, if one is specifically looking for these signs and finds some of them but not others, the positive results are much less likely actually to mean that the assumptions are confirmed. Especially given the sensitivity of peculiar velocity studies to the background model as shown above, the standard of proof must be set higher than the occasional positive result. If it looks like a duck, but doesn't quack quite like a duck or fly at all like a duck, it probably isn't a duck. (It may be a decoy.) It is certainly possible that, for instance, gravitating mass might be found near a visible galaxy group now and then by chance.

Note that we do not know the background against which peculiar velocity studies should take place. We have an average Hubble constant and solar reflex velocity computed from the data; these are statistics, which we hope approximate the real background well enough. Similarly, the uncertainties in background model parameters (and hence peculiar velocities) are based on how well the model fits the data at hand; they are not absolute.

That said, there have been previous indications that galaxy groups in the Local Volume do not have the expected gravitational effect on peculiar velocities. Karachentsev et al. (2003a) found the Canes Venatici Group's kinematics to be mostly a matter of Hubble expansion, as did Jerjen et al. (1998) for the Sculptor Group (the latter result has been supported by other studies). Whiting (2003) found that there was no significant difference in velocity dispersion within the Local Volume between galaxies within groups and field galaxies.

\subsection{Results on Other Scales}

The main result of this study also appears to be at odds with some results on smaller and larger scales. The venerable timing argument (whether in the original two-body form of Kahn \& Woltjer (1959) or with dwarf galaxies added, as in Whiting (1999) for instance) gives reasonable results for the mass and age of the Local Group; the more sophisticated Least-Action approach of Peebles (1990) (among other implementations) also seems to work well. On larger scales, as has been noted, there is agreement between the luminosity and inferred mass fields; in addition, a version of the Least Action reaching down to Local Volume scales has been successful here (Sharpe et al. 2001). While I have been careful to note that 
the present work does not directly apply to other scales, it is worth looking at how much of a disagreement there is and how it might be resolved.

There is the possibility of a fortuitous result, for instance that a mass of dark matter is located by chance in such a way as to produce motion consistent with light tracing mass in one area, while such a condition is not general in the Volume. A good result with the Local Group then becomes a matter of small-number (one) statistics.

Small-number statistics can also enter in when checking how good a result is. Both Peebles (1990) and Sharpe et al. (2001) use a few galaxies (six and twelve, respectively) to test their fits. For comparison, Whiting (2003) showed that the fourteen galaxies used by Ekholm et al. (2001) to characterize Local Volume motions gave a quite misleading picture. The same thing could be happening here (and note that one of the galaxies in Sharpe et al. (2001) did not fit well at all).

But mostly it appears that there are differences in interpretation as to what constitutes a good result. The timing argument produces a number in which the age and mass of the Local Group are mixed. Given an age in accordance with other determinations, one finds a mass which is in reasonable agreement with other determinations. But the latter is not really known independently to within a factor of two (or more), so the timing argument produces am age consistent with other results only within this factor. Moreover, as the plot in Whiting (1999) shows, there is a large uncertainty (certainly another factor of two) in the combined age-mass number. The best that can be said is that the timing argument shows that its assumptions produce a result which is roughly consistent with other determinations.

The calculations of Peebles (1990) and Sharpe et al. (2001) produce much tighter correlations between calculated and observed redshifts for their test galaxies. But if one examines instead the correlation between predicted and observed peculiar velocities, the correlation looks much looser, to the point where it might not be significant in the context of a $75 \mathrm{~km} \mathrm{~s}^{-1} \mathrm{rms}$ dipersion. Probably the best interpretation is that these studies have arrived at the right overall density of mass, as well as a good approximation of fluctuation amplitude; but (in light of the present work) may not have located those fluctuations correctly.

\subsection{Conclusions and Implications}

Given the overall result that the peculiar velocity field and the luminosity field do not correlate, one of two conclusions follow:

- Either light does not trace mass in the Local Volume, or 
- Peculiar velocities are not produced by gravitational interaction.

Just how light might fail to trace mass has not been shown in any detail. There could be a large population of "dark galaxies", such as those postulated by Jimenez et al. (1997) on the basis of lensed quasars. Attempts to reconstruct the local dark matter field from the peculiar velocity field using a POTENT-type algorithm (see, for example, Bertschinger et al. (1990)) have not been successful. It appears that 149 data points are just too few (POTENT calculations use galaxies in the thousands). It does appear that theories which dispense with dark matter by modifying the behavior of gravity are ruled out by this result.

It is even less obvious how peculiar velocities might be produced if gravitational interaction is not the source. The only competing explanation, primeval turbulence, seems to have been ruled out long ago (see Peebles (1993), pp. 541ff).

This result does not mean that mass and light are unrelated on larger scales (indeed, as noted in the Introduction, they seem to go together well there). Nor does it mean that they are unrelated on galaxy scales, where clearly dark matter haloes are concentric with luminous matter. Nor does it mean that they are unrelated on Mpc scales in galaxy clusters, where again they seem to be concentric. It does mean that a plausible and convenient assumption commonly made must be abandoned; but in return we have gained some important clues as to the behavior (and, one hopes eventually, the nature) of this elusive dark matter.

The author gratefully acknowledges help from discussions with many collegues, especially Dr. Donald Lynden-Bell, Dr. Nicholas B. Suntzeff and Dr. David Spergel; and would like to express his sincere appreciation for all the observers whose data appear in Table (1).

This research has made use of the NASA/IPAC Extragalactic Database (NED) which

is operated by the Jet Propulsion Laboratory, California Institute of Technology, under contract with the National Aeronautics and Space Administration.

\section{REFERENCES}

Aparicio, A., Dalcanton, J. J., Gallart, C. \& Martínez-Delgado, D. 1997, AJ, 114, 1447

Aparicio, A., \& Tikhonov, N. 2000, AJ, 119, 2183

Aparicio, A., Tikhonov, N., \& Karachentsev, I. 2000, AJ, 119, 177

Armandroff, T. E., DaCosta, G. S., Caldwell, N., \& Seitzer, P. 1993, AJ, 106, 986 
Bellazzini, M., Ferraro, F. R., Origlia, L., Pancino, E., Monaco, L., \& Oliva, E. 2002, AJ, 124,3222

Bertschinger, E., Dekel, A., Faber, S. M., Dressler, A., \& Burstein, D. 1990, ApJ, 364, 370

Bonanos, A. Z., Stanek, K. Z, Szentgyorgyi, A. H., Sasselov, D. D., \& Bakos, G. Á. 2004, AJ, 127, 861

Branchini, E., Zehavi, I., Plionis, M., \& Dekel, A. 2000, MNRAS, 313, 491

Cannon, J. M., Dohm-Palmer, R. C, Skillman, E. D., Bomans, D. J., Côté, S., \& Miller, B. W. 2003, AJ, 126, 2806

Clementini, G., Held, E. V., Baldacci, L., \& Rizzi, L. 2003, ApJ, 588, 85

Cole, A. A., et al. 1999, AJ, 118, 1657

Courteau, S., Strauss, M. A., \& Willick, J. A. 2000, Cosmic Flows 1999 (ASP Conference Series \#201; San Franciso: Astronomical Society of the Pacific)

Crone, M. M., Schulte-Ladbeck, R. E., Hopp, U., \& Greggio, L. 2000, ApJ, 545, L31

DaCosta, G. S., Armandroff, T. E., Caldwell, N., \& Seitzer, P. 2000, AJ, 119, 705

Davidge, T. J. 2003, AJ, 125, 3046

Dohm-Palmer, R. C., et al. 1998, AJ, 116, 1227

Dolphin, A. E. et al. 2001, ApJ, 550, 554

Dolphin, A. E. et al. 2001, MNRAS, 324, 249

Dolphin, A. E. et al. 2002, AJ, 123, 3154

Dolphin, A. E. et al. 2003, AJ, 125, 1261

Drosdovsky, I. O., \& Karachentsev, I. D. 2000, A\&AS, 142, 425

Drosdovsky, I. O., Schulte-Ladbeck, R. E., Hopp, U., Crone, M. M., \& Greggio, L. 2001, ApJ, 551, L135

Drozdovsky, I. O., Schulte-Ladbeck, R. E., Hopp, U., Greggio, L., \& Crone, M. M. 2002, AJ, 124,811

Ekholm, T., Baryshev, Yu., Terrikorpi, P., Hanski, M. O., \& Paturel, G. 2001, A\&A, 368, L17 
Fingerhut, R. L., McCall, M. L, de Robertis, M., Kingsburgh, R. L, Komljenovic, M., Lee, H., \& Buta, R. J. 2003, ApJ, 587, 672

Freedman, W. L. et al. 2001, ApJ, 553, 47

Freeman, K. C., Karlsson, B., Lyngå, G., Burrell, J. F., van Woerden, H., Goss, W. M., \& Mebold, U. 1977, A\&A, 55, 445

Frogel, J. A., Persson, S. E, Aaronson, M., \& Matthews, K. 1978, ApJ, 220, 75

Gallagher, J. S., Tolstoy, E., Dohm-Palmer, R. C., Skillman, E. D., Cole, A. A., Hoessel, J. G., Saha, A, \& Mateo, M. 1998, AJ, 115, 1869

Gallart, C., Martínez-Delgado, D., Gómez-Flechoso, M. A., \& Mateo, M. 2001, AJ, 121, 2572

Han, M., et al. 1997, AJ, 113, 1001

Harries, T. J., Hilditch, R. W., \& Howarth, I. D. 2003, MNRAS, 339, 157

Held, E. V., Savine, I., \& Momany, Y. 1999, A\&A, 345, 747

Herrnstein, J. R., Moran, J. M., Greenhill, L. J., Diamond, P. J., Inoue, M., Nakai, N., Miyoshi, M., Henkel, C. \& Riess, A. 1999, Nature, 400, 539

Hidalgo, S. L., Marín-Franch, A., \& Aparicio, A. 2003, AJ, 125, 1247

Hoessel, J. G., Saha, A., \& Danielson, G. E. 1998, AJ, 115, 573

Hoessel, J. G., Saha, A., \& Danielson, G. E. 1998, AJ, 116, 1679

Hopp, U., Schulte-Ladbeck, R. E., Greggio, L., \& Mehlert, D. 1999, A\&A, 342, 19

Huchtmeier, W. K., Karchentsev, I. D., Karachentseva, V. E., \& Ehle, M. 2000, A\&A Sup., 141,469

Huchtmeier, W. K., Karachentsev, I. D., \& Karachentseva, V. E. 2000, A\&A Sup., 147, 187

Hunter, D. A. 2001, ApJ, 559, 225

Irwin, M., \& Tolstoy, E. 2002, MNRAS, 336, 643

Izotov, Y. I., \& Thuan, T. X. 2002, ApJ, 567, 875

Jerjen, H., Freeman, K. C., \& Binggeli, B. 1998, AJ, 116, 2873 
Jerjen, H., \& Rejkuba, M. 2001, A\&A, 371, 487

Jimenez, R., Heavens, A. F., Hawkins, M. R. S., \& Padoan, P. 1997, MNRAS, 292, L5

Kahn, F. D., \& Woltjer, L. 1959, ApJ, 130, 705

Karachentsev, I. D., \& Makarov, D. A. 1996, AJ, 111, 794

Karachentsev, I. D., Aparicio, A., \& Makarova, L. 1999a, A\&A, 352, 363

Karachentsev, I. D., Sharina, M. E., \& Huchtmeier, W. K. 2000a, A\&A, 362, 544

Karachentsev, I. D., et al. 2000b, A\&A, 363, 117

Karachentsev, I. D., \& Makarov, D. A. 2001, Astrophysics, 44, 1

Karachentsev, I. D. et al. 2001a, A\&A, 379, 407

Karachentsev, I. D. et al. 2002a, A\&A, 383, 125

Karachentsev, I. D. et al. 2002b, A\&A, 385, 21

Karachentsev, I. D. et al. 2002c, A\&A, 389, 812

Karachentsev, I. D. et al. 2003a, A\&A, 398, 467

Karachentsev, I. D. et al. 2003b, A\&A, 398, 479

Karachentsev, I. D. et al. 2003c, A\&A, 404, 93

Karachentsev, I. D., Sharina, M. E., Dolphin, A. E., \& Grebel, E. K. 2003d, A\&A, 408, 111

Karachentsev, I. D., Karachentseva, V. E., Huchtmeier, W. K., \& Makarov, D. I. 2004, AJ, 127,2031

Kent, S. M., Dame, T. M., \& Fazio, G. 1991, ApJ, 378, 131

Kerr, F. J., \& Lynden-Bell, D. 1986, MNRAS, 221, 1023

Klypin, A., Hoffman, Y., Kravtsov. A. V., \& Gottlöber, S. 2003, ApJ, 596, 19

Kunkel, W. E., \& Demers, S. 1977, ApJ, 214, 21

Lee, M. G., Freedman, W. L., \& Madore, B. F. 1993, AJ, 106,964

Lee, M. G., \& Byun, Y.-I. 1999, AJ, 118, 817 
Lee, M. G., Aparicio, A., Tikhonov, N., Byun, Y.-I., \& Kim, E. 1999, AJ, 118, 853

Lee, M. G., Kim, M., Sarajedini, A., Geisler, D., \& Gieren, W. 2002, ApJ, 565, 959

Lee, M. G., et al. 2003, AJ, 126, 2840

Lynds, R., Tolstoy, E., O’Neill jr., E. J, \& Hunter, D. A. 1998, AJ, 116, 146

Mackey, A. D., \& Gilmore, G. 2003, MNRAS, 343, 747

Maíz-Apellániz, J., Cieza, L., \& MacKenty, J. W. 2002, AJ, 123, 1307

Makarova, L. N., \& Karachentsev, I. D. 2003, Astrofizika, 46, 144

Mateo, M., Olszewski, E. W., Pryor, C., Welch, D. L., \& Fischer, P. 1993, AJ, 105, 510

Méndez, B., Davis, M., Moustakas, J., Newman, J., Madore, B. F., \& Freedman, W. L. 2002, AJ, 124, 213

Martínez-Delgado, D., Gallart, C., \& Aparicio, A. 1999, AJ, 118, 862

McConnachie, A. W., Irwin, M. J., Ferguson, A. M. N., Ibata, R. A., Lewis, G. F., \& Tanvir, N. 2004, MNRAS, 350, 243

Mighell, K. J. 1997, AJ, 114, 1458

Mihalas, D., \& Binney, J. 1984, Galactic Astronomy (San Francisco: W. H. Freeman)

Miller, B. W., Dolphin, A. E., Lee, M. G., Kim, S. C. \& Hodge, P. 2001, ApJ, 562, 713

Minniti, D., \& Zijlstra, A. A. 1997, AJ, 114, 147

Mould, J., Kristian, J., \& DaCosta, G. S. 1984, ApJ, 278, 575

Musella, I., Piotto, G., \& Capaccioli, M. 1997, AJ, 114, 976

Nusser, A., da Costa, L. N., Branchini, E., Bernardi, M., Alonso, M. V., Wegner, G., Willmer, C. N. A., \& Pelligrini, P. S. 2001, MNRAS, 320, L21

Peebles, P. J. E. 1990, ApJ, 362,1

Peebles, P. J. E. 1993, Principles of Physical Cosmology (Princeton: Princeton University Press)

Prada, F., et al. 2003, ApJ, 598, 260 
Pritzl, B. J., Armandroff, T. E., Jacoby, G. H., \& Da Costa, G. S. 2002, AJ, 124, 1464

Pritzl, B. J., Armandroff, T. E., Jacoby, G. H., \& Da Costa, G. S. 2004, AJ, 127, 318

Puche, D., \& Carignan, C. 1988, AJ, 95, 1025

Rawson, D. M., et al. 1997, ApJ, 490, 517

Rejkuba, M. 2004, A\&A, 413, 903

Saha, A., Claver, J., \& Hoessel, J. G. 2002, AJ, 124, 839

Sakai, S., \& Madore, B. F. 1999, ApJ, 526, 599

Sakai, S., Madore, B. F., \& Freedman, W. L. 1997, ApJ, 480, 589

Sakai, S., Madore, B. F., \& Freedman, W. L. 1999, ApJ, 511, 671

Sakai, S., \& Madore, B. F. 2001, ApJ, 555, 280

Saviane, I., Held, E. V., \& Piotto, G. 1996, A\&A, 315, 40

Sharina, M. E., Karachentsev, I. D., \& Tikhonov, N. A. 1996, A\&AS, 119, 499

Sharina, M. E, Karachentsev, I. D., \& Burenkov, A. N. 2001, A\&A, 380, 435

Sharpe, J., et al. 2001, MNRAS, 322, 121

Shlegel, D. J., Finkbeiner, D. P., \& Davis, M. 1998, ApJ, 500, 525

Stanek, K. Z., \& Garnavich, P. M. 1998, ApJ, 503, 131

Stetson, P. B. et al. 1998, ApJ, 508, 491

Suntzeff, N. B., Schommer, R. A., Olszewski, E. W., \& Walker, A. R. 1992, AJ, 104, 1743

Thim, F., Tammann, G. A., Saha, A., Dolphin, A., Sandage, A., Tolstoy, E. \& Labhardt, L. 2003, ApJ, 590, 256

Thim, F., Hoessel, J. G., Saha, A. Claver, J., Dolphin, A. \& Tammann, G. A. 2004, AJ, 127, 2322

Tikhonov, N. A., Galazutdinova, O. A., \& Aparicio, A. 2003, A\&A, 401, 863

Tonry, J.L., Dressler, A., Blakeslee, J. P., Ahjar, E. A., Fletcher, A. B., Luppino, G. A., Metzger, M. R., \& Moore, C. B. 2001, ApJ, 546, 681 
Tolstoy, E., 1995, PhD thesis, Univ. of Groningen (cited in Dohm-Palmer et al. (1998))

Tolstoy, E. et al. 1998a, AJ, 116, 1244

Tolstoy, E., \& Irwin, M. MNRAS, 318, 1241

Tolstoy, E., Irwin, M., Cole, A., Fraternali, F., Szeifert, T., \& Marconi, G. 2004, ESO Messenger \# 115, 32

Tosi, M., Sabbi, E., Bellazzini, M., Aloisi, A., Greggio, L., Leitherer, C., \& Montegriffo, P. 2001, AJ, 121, 1271

Tully, R. B., Boesgaard, A. M., Dyck, H. M., \& Schempp, W. V. 1981, ApJ, 246, 38

Whiting, A. B. 1999, in IAU Symposium no. 192, The Stellar Content of Local Group Galaxies, eds. P. Whitelock \& R. Cannon (San Francisco: ASP), 420

Whiting, A. B. 2003, AJ, 587, 186

Wyder, T. K. 2003, AJ, 125, 3097

Yahil, A., Tammann, G. A., \& Sandage, A. 1977, ApJ, 217, 903

Young, L. M., van Zee, L., Lo, K.Y., Dohm-Palmer, R. C., \& Beierle, M. 2003, ApJ, 592, 111

Zijlstra, A. A., \& Minniti, D. 1999, AJ, 117, 1743 